\title{
Comparative review of theoretical models for elastic wave attenuation and dispersion in partially saturated rocks
}

\author{
J. Toms ${ }^{\mathrm{a}^{*}}$, T. Müller ${ }^{\mathrm{b}}$, R. Ciz ${ }^{\mathrm{c}}$ and B. Gurevich ${ }^{\mathrm{a}, \mathrm{c}}$ \\ ${ }^{a}$ Dept. Exp. Geophys., Curtin University of Technology, Australia \\ ${ }^{b}$ Geophysikalisches Institut, Uni Karlsruhe, Germany \\ ${ }^{c}$ CSIRO Petroleum, Australia
}

\begin{abstract}
Saturation of porous rocks with a mixture of two fluids (known as partial saturation) has a substantial effect on the seismic waves propagating through these rocks. In particular, partial saturation causes significant attenuation and dispersion of the propagating waves, due to wave-induced fluid flow. Such flow arises when a passing wave induces different fluid pressures in regions of rock saturated by different fluids. As partial fluid saturation can occur on different length scales, attenuation due to wave induced fluid flow is ubiquitous. In particular, mesoscopic fluid flow due to heterogeneities occurring on a scale greater than porescale, but less than wavelength scale, is responsible for significant attenuation in the frequency range from 10 to $1000 \mathrm{~Hz}$.
\end{abstract}

Most models of attenuation and dispersion due to mesoscopic heterogeneities imply that fluid heterogeneities are distributed in a periodic/regular way. In $1 \mathrm{D}$ this corresponds to periodically alternating layering, in 3D as periodically distributed inclusions of a given shape (usually spheres). All these models yield very similar estimates of attenuation and dispersion.

\footnotetext{
* Corresponding author: Julianna.Toms@geophy.curtin.edu.au, Curtin University of Technology, GPO Box U1987, Perth, Western Australia 6845, Ph +61 892663521 Fax Number 61892663407
} 
Experimental studies show that mesoscopic heterogeneities have less idealised distributions and that the distribution itself affects attenuation and dispersion. Therefore, theoretical models are required which would simulate the effect of more general and realistic fluid distributions.

We have developed two theoretical models which simulate the effect of random distributions of mesoscopic fluid heterogeneities. The first model assumes that one fluid forms a random ensemble of spherical inclusions in a porous medium saturated by the other fluid. The attenuation and dispersion predicted by this model are very similar to those predicted for 3D periodic distribution. Attenuation (inverse quality factor) is proportional to $\omega$ at low frequencies for both distributions. This is in contrast to the $1 \mathrm{D}$ case, where random and periodically alternating layering shows different attenuation behaviour at low frequencies. The second model, which assumes a 3D continuous distribution of fluid heterogeneities, also predicts the same low-frequency asymptote of attenuation. However, the shapes of the frequency dependencies of attenuation are different. As the 3D continuous random approach assumes that there will be a distribution of different patch sizes, it is expected to be better suited to modelling experimental results. Further research is required in order to uncover how to relate the random functions to experimentally significant parameters.

Keywords: Poroelasticity; Patchy saturation; Mesoscopic flow; Attenuation; Phase velocity dispersion 


\section{Introduction}

Partial saturation of porous rock by two or more different fluids can occur in a multitude of geological settings. For instance, gas, oil and brine commonly share the available pore space in the upper part of gas capped reservoirs. Underground aquifers can become infiltrated by contaminating fluids. Earthquake events can induce ground water variations and inturn; after shocks have been linked to changes in pore fluid distribution. In order to better assist the interpretation of seismic data acquired for the purposes of detecting hydrocarbons, monitoring or tracking saltwater intrusions into ground water aquifers, or for analyzing recorded waveforms from earthquake events, knowledge of how partial fluid saturation affects elastic wave propagation is required.

The propagation of elastic waves in fluid saturated porous media is usually described by Biot's equations of poroelasticity [1-3]. In the low-frequency (static) limit these equations yield the so-called Gassmann equation [4], which expresses the undrained static bulk modulus of the porous medium as a function of the properties of the dry frame and the saturating fluid. Both Biot and Gassmann equations assume that the porous medium is saturated with a single Newtonian fluid (liquid or gas).

Extending Biot-Gassmann theory to model wave propagation in porous media saturated by two or more immiscible fluids is not trivial. Immiscibility implies that the fluids are not dissolved into one another and a distinct fluid-fluid interface exists which separates each fluid [5]. When two immiscible fluids are distributed on a relatively fine scale, they can be regarded as a single composite fluid whose compressibility (inverse of bulk 
modulus) is given by an average of its constituent compressibilities (using the so-called Wood equation [6]). In this circumstance, Gassmann's equation can be applied to determine an effective bulk modulus of the porous medium, in which the fluid bulk modulus is now given by the composite average. This case is often referred to as uniform saturation, and implies full pressure equilibration between the two fluids.

This pressure equilibration can only be achieved if the frequency is sufficiently low so that the characteristic length of fluid diffusion in the pore space is large compared to the largest spatial scale of fluid mixing. If the frequency is higher, the pressure in the two fluids will not have enough time to equilibrate, resulting in a higher undrained bulk modulus and wave velocity. Hence, the presence of two fluids in the pores (so called partial saturation) causes an additional dispersion and attenuation of elastic waves, which is related to relaxation of pore fluid pressures. The frequency dependency of wave velocity and attenuation in a partially saturated medium is controlled by the size, shape and spatial distribution of fluid pockets and permeability and elastic moduli of the solid matrix as well as the properties of the two fluids.

In the last 30 years a numbers of models have been introduced that correspond to different spatial configurations of fluid pockets. Most of these models assume regular fluid patterns such as a cubic lattice of gas pockets of a fixed shape in a liquid-saturated background medium [7-9]. However, spatially regular distribution of the fluids may not always give an adequate representation of the real distribution. Moreover, it has been shown $[10,11]$ that random and periodic 1D distributions of two different fluids yield 
very different attenuation/dispersion pairs. Although 1D alternating fluid distributions may not be realistic, this result gives an additional motivation to studies of wave propagation in porous media with random spatial fluid distributions. Recent results show that this approach is promising $[12,13]$.

The aim of this paper is to provide a review of the different approaches to modelling wave propagation in partially fluid saturated porous media. First, a brief summary of the Biot-Gassmann theory of poroelasticity will be given. The next section will review models for partial fluid saturation, which utilize regular spatial fluid patterns, followed by our recent models, which utilize a random fluid distribution. Finally, some numerical examples will be given, which compare attenuation and dispersion estimates using the different modelling approaches.

\section{Elastic wave propagation in fully fluid saturated porous media}

Biot's equations of dynamic poroelasticity [1-3] provide a general framework for modelling elastic wave propagation through porous fluid saturated media. The equations were derived using a Lagrangian view point with generalised coordinates given by the average solid and fluid displacements. A dissipation function was introduced, which depended only upon relative solid and fluid motion. Subsequently, Biot's equations have been rederived using a number of different mathematical techniques, such as volume averaging methods [14] and homogenization for periodic structures [15-17]. All of these methods yield exactly the same macroscopic equations, thus confirming the validity of Biot's original formulation. 
The basic assumptions of Biot's equations [1-3] are:

I) The porous rock frame is homogenenous and isotropic. It has uniform porosity $\phi$, bulk modulus $K_{d}$, shear modulus $\mu_{0}$, density $\rho_{0}$ and permeability $\kappa$, and consists of only one grain type, characterized by bulk modulus $K_{g}$, shear modulus $\mu_{g}$ and density $\rho_{g}$.

II) The porous rock is fully saturated by only one fluid having viscosity $\eta$, fluid bulk modulus $K_{f}$, and density $\rho_{f}$.

III) Relative motion between solid and fluid is governed by Darcy’s law.

IV) The wavelength of the passing wave is substantially larger than the size of the largest grains or pores.

The Biot wave equations describing average solid $\mathbf{u}$ and fluid displacement $\mathbf{U}$ can be written in the frequency domain (with time dependence exp $(-i \omega t)$ implied) as [3]

$(H-\mu) \operatorname{grad} \operatorname{div} \mathbf{u}+\mu \nabla^{2} \mathbf{u}+\alpha M \operatorname{grad} \operatorname{div} \mathbf{w}+\omega^{2}\left(\rho \mathbf{u}+\rho_{f} \mathbf{w}\right)=0$,

$\alpha M \operatorname{grad} \operatorname{div} \mathbf{u}+M \operatorname{grad} \operatorname{div} \mathbf{w}+\omega^{2}\left(\rho_{f} \mathbf{u}+q \mathbf{w}\right)=0$,

where $\mathbf{w}=\phi(\mathbf{U}-\mathbf{u})$ represents the average fluid displacement relative to the solid, $\rho=(1-\phi) \rho_{g}+\phi \rho_{f}$ is the density of the porous fluid-saturated rock, $\alpha=1-K_{d} / K_{g}$ is so called the Biot-Willis coefficient [18], and $H, \mu$, and $M$ are material properties defined 
later in this section. Parameter $q(\omega)$ is a frequency dependent coefficient responsible for viscous and inertial coupling between the solid and fluid motion, and is given by

$q=\rho_{f}\left[\frac{\chi}{\phi}+\frac{i \eta}{\kappa^{*}(\omega) \omega \rho_{f}}\right]$.

Here $\chi \leq 1$ is the tortuosity, a dimensionless parameter which is responsible for inertial coupling between solid and fluid motion. The parameter $\kappa^{*}$ is the dynamic hydraulic permeability, which in general, is frequency dependent and responsible for viscous coupling. For sufficiently low frequencies (lower than Biot's characteristic frequency $\left.f_{c}=\phi \eta /\left(2 \pi \kappa \rho_{f}\right)\right)$ fluid flow within the pore channels is approximated as Poiseuille. This means that the flow is laminar (ie the Reynolds number of the flow which expresses the ratio of inertial forces to viscous forces is less than a critical Reynolds number [5]). In this case the first bracketed term in the right-hand side of (3) can be neglected [19] and the dynamic permeability reduces to the steady-state permeability $\kappa$, giving

$q=\frac{i \eta}{\kappa \omega}$.

For most rocks and soils Biot's characteristic frequency $f_{c}$ turns out to be about $10^{5} \mathrm{~Hz}$ or higher. Therefore, for most seismic applications the low-frequency version of Biot theory is adequate. 
For a homogeneous porous medium equations (1) and (2) form a system of six linear partial differential equations with constant coefficients for six components of two vectorfunctions $\mathbf{u}$ and $\mathbf{w}$. By considering a solution of these equations dependent upon only one coordinate, say $x$, we can reduce equations (1) and (2) to a system of six second-order linear ordinary differential equations with one independent variable $x$. The equations for $u_{y}, u_{z}, w_{y}$, and $w_{z}$ describe the propagation of two identical shear modes with two orthogonal polarisations. These shear waves are very similar in nature to classical shear waves in an isotropic viscoelastic medium. The remaining system of two equations for $u_{x}, w_{x}$ has a solution of the form

$\left(\begin{array}{l}u_{x} \\ w_{x}\end{array}\right)=\left(\begin{array}{l}u_{x 0} \\ w_{x 0}\end{array}\right) \exp (i k x)$,

where wavenumber $k$ is the root of its characteristic equation, that is, an eigenvalue of the linear algebraic system obtained by substituting (5) into (1) and (2). The characteristic equation is quadratic in $k^{2}$, and thus yields two pairs of complex roots $\pm k_{+}$ and $\pm k_{-}$. This shows that in a porous medium there exist two types of compressional waves with complex velocities $v_{+,-}=\omega / \operatorname{Re}\left\{k_{+,-}\right\}$and attenuation factors (inverse quality factors) $Q_{+,-}^{-1}=\operatorname{Im}\left\{k_{+,-}^{2}\right\} / \operatorname{Re}\left\{k_{+,-}^{2}\right\}$.

The compressional waves are termed fast (+) and slow (-) $P$-waves and occur when solid and fluid particle motion is in phase or out of phase, respectively. The fast wave is a direct analog of the normal compressional wave in an elastic or viscoelastic solid, it 
exhibits small amounts of attenuation and phase velocity dispersion (see Figure 1). On the other hand, the slow $P$ - wave behaves very differently at low and high frequencies. At low frequencies $f<<f_{c}$, the wavenumber of the slow $P$ wave is given by $k_{-}^{2}=i \omega \eta / \kappa N$ where $N=M L / H$. In this frequency regime, the slow $P$ wave is highly attenuated and is analogous to diffusion or heat conduction. On the other hand, at high frequencies $f>f_{c}$ the slow $P$-wave is propagatory with the propagation velocity approaching $c_{f} \chi^{-1 / 2}$, where $c_{f}$ is sound velocity in the free fluid.

For frequencies less than Biot's characteristic frequency, the fast $P$ and shear wave numbers are given by [20]

$k_{+}^{2}=\frac{\omega^{2}}{v_{+}^{2}}\left(1+\frac{i}{Q_{+}}\right), k_{s}^{2}=\frac{\omega^{2}}{v_{s}^{2}}\left(1+\frac{i}{Q_{s}}\right)$,

where $v_{+}=\sqrt{H / \rho}$ is the fast $P$ wave velocity and $v_{s}=\sqrt{\mu / \rho}$ is the shear wave velocity. In this frequency range the attenuation (inverse quality factors) for fast $P$ and shear waves are $Q_{+}^{-1}=\omega \kappa \rho_{f}\left(1-v_{0}^{2} / v_{+}^{2}\right)^{2} / \eta \rho$, and $Q_{s}^{-1}=\omega \kappa \rho_{f} / \eta \rho$, where $v_{0}=\sqrt{\alpha M / \rho_{f}}$.

Parameter $H$ that appears in the expression for the fast $P$ wave velocity $v_{+}$is called the saturated $P$ wave modulus, and can be written as $H=K+4 \mu / 3$, where $K$ and $\mu$ are the undrained bulk and shear moduli of the fluid-saturated porous medium given by the equations:

$$
\begin{aligned}
& K=K_{d}+\alpha^{2} M, \\
& M=\left[(\alpha-\phi) / K_{g}+\phi / K_{f}\right]^{-1},
\end{aligned}
$$


$\mu=\mu_{0}$,

where the analogous $\mathrm{P}$ wave modulus for a dry medium is given by $L=K_{d}+4 \mu_{0} / 3$.

Equations (7) - (9) have first been derived by Gassmann [4] and are referred to as Gassmann equations. For Gassmann equations to be applicable, several conditions must be met. The pore space within the rock must be connected so pore fluid can achieve equilibration. Thus, fluid pressure effects due to isolated pore spaces are not accounted for. Furthermore, the frequency must be sufficiently low, so that, fluid pressures induced by a passing wave have enough time for pressure equilibration.

In essence, Gassmann equations define elastic wave velocities in fluid-saturated porous media in the low frequency limit. These equations are widely used in the petroleum industry for estimating seismic wave velocities in hydrocarbon reservoirs [21,22]. However, in general, seismic wave propagation often violates the quasi-static assumption, causing deviations from Gassmann results. In particular, wave attenuation and phase velocity dispersion cannot be modelled with Gassmann equation. To account for these effects Biot theory is often utilized.

Wave attenuation and phase velocity dispersion within Biot type media is caused by global or macroscopic fluid flow, which is called "Biot loss". It occurs when pore fluids develop spatial gradients in fluid pressure induced over the wavelength of an incident compressional wave. This drives fluid flow relative to the rock frame, causing wave energy to be lost through viscous dissipation. 
Although Biot theory provides a mechanism for the dissipation and dispersion of elastic waves, it is generally accepted that it cannot adequately explain observed magnitudes of attenuation and dispersion, especially within the low frequency regime [23-26]. However, it is widely accepted that Biot theory is correct in predicting the existence of the slow $P$ wave. It has been confirmed by a number of laboratory experiments [27-29].

\section{Partially fluid saturated porous media}

Partial fluid saturation of porous rock by multiple types of pore fluids was first proposed as a cause for the mismatch between experimental measurements of attenuation and phase velocity dispersion, and theoretical predictions given by Biot theory [1-3]. White $[7,30]$ was the first to show theoretically that partial fluid saturation can cause significant attenuation and phase velocity dispersion. Experimental studies conducted around the same time [31,32] also indicated that partial fluid saturation of porous rock exhibits different phase velocity behaviour then fully saturated porous rock.

Since then, the study of elastic wave propagation in partially fluid saturated media has become a field of interest in its own right, generating a number of experimental $[25,33$ 35], numerical [37-41] and theoretical studies designed to elucidate key features, which cause attenuation and phase velocity dispersion. 
There are a number of different approaches to theoretically modelling attenuation and dispersion due to the presence of partial fluid saturation. Each approach emphasizes a particular physical aspect, thought to significantly affect attenuation and dispersion estimates. Broadly speaking, most approaches focus on:

I) Pore scale distribution of immiscible fluids: These models are often called local or "squirt" flow models [42-45]. Attenuation and phase velocity dispersion arise due to fluid flow occurring between gas and liquid filled areas of the same pore or crack.

II) Mesoscale distribution of immiscible fluids: fluid heterogeneities occur on the scale greater than the pore scale, but less than wavelength scale. Fluid heterogeneities can be modelled using a periodic distribution $[7-9,30]$ or a random distribution [11-13]. Attenuation and phase velocity dispersion arises due to induced pressure gradients on the mesoscale, which causes fluid to flow.

III) Defining an effective pore fluid: incorporating free bubble oscillations [46-49]. Attenuation can arise due to viscous and thermal damping, which occurs when the free gas bubble oscillates in response to pressure fluctuations in the surrounding pore liquid.

There are many different types of porous rocks, such as sandstones, limestones, shales etc, which are often saturated by different combinations of pore fluids, such as water, oil and gas. As such, in some situations, one theoretical approach may be more applicable than another. 
Category I models are good for situations where porous rocks are known to contain a large number of very compliant grain contacts or cracks. This becomes especially important for laboratory studies where rock samples have undergone distortion due to removal from in situ conditions, which can either induce fracturing on the grain scale or cause the opening of otherwise closed grain contacts, due to changes in confining pressure [9]. Furthermore, certain rock samples or in situ rocks which contain significant grain-scale heterogeneities are also suitable, such as with carbonate rocks which possess both intergranular and intragranular porosity [50] or for sandstones having either imperfectly cemented grain contacts [44] or an assemblage of smaller irregularly shaped intra-pore minerals [51].

In those circumstances, wetting fluids like water preferentially saturate grain contacts and cracks, whilst non-wetting fluids like gas assume larger rounder pore spaces [44]. In response to a passing wave, spatial gradients in fluid pressure develop which cause fluid to flow between grain cracks, contacts etc and rounder pore spaces. This occurs because cracks, contacts etc are more compliant (mechanically weaker) than rounder pore spaces.

Fluid flow on the pore scale also known as local (or squirt) flow causes attenuation and phase velocity dispersion. The reason is that when wave frequencies are sufficiently low there is enough time for fluid to flow, whilst at higher frequencies there isn't. This means that a porous rock at lower frequencies is less stiff than at higher frequencies resulting in lower wave velocities. At intermediate wave frequencies, phase velocity is frequency dependent and attenuation is a maximum. 
A possible limitation of most squirt flow models is that they assume specific pore scale geometries. In particular, Murphy [44] assumes that the grain contact is perfectly flat and adjacent to a spherical pore, whilst Mavko and Nur [42] model a multitude of idealised geometries, such as flat, triangular and parabolic pore shapes. Thus appropriate application of these models requires knowledge of rock characteristics on the porescale, which isn't always available, certainly for in situ applications.

As it is well known that bubbles affect the acoustic properties of a liquid [52-54], category III models are best suited to applications where fluid-fluid interaction is considered important. In a free liquid the presence of bubbles has two interconnected effects on the acoustic properties of the liquid, which can also affect wave propagation when that fluid saturates porous rock. Firstly, the presence of bubbles affects the compressibility of the liquid; this changes the propagation velocity of waves. Secondly, pressure fluctuations within the liquid stemming from wave propagation, forces bubbles to oscillate about their equilibrium radius. This causes attenuation as wave energy is transferred into energy which drives bubble oscillations.

On the other hand, category II models are best suited to applications where wave frequencies are low and the porous rock is saturated by relatively large (mesoscopic) fluid patches. For the rest of the review, we will focus only on category II models; however we wish to acknowledge the importance of both category I and III models. 


\subsection{Mesoscopic distribution of fluids}

Fluid heterogeneities existing on a scale which is greater than pore scale, but less than wavelength scale are called mesoscopic. A mesoscopic distribution of two pore fluids can arise due to variations in porosities, permeabilities and grain types within a porous rock. These features will cause pore fluids to be preferentially located in different positions, e.g., in a way shown in Figure 2.

On the pore scale, numerical studies [55-59] have shown that water preferentially locates in grain contacts and smaller pore spaces, whilst gas prefers larger rounder pore spaces. Presumably, the same physics which dictates fluid distribution on the pore scale, such as minimization of interfacial surface area, between grains and fluids, and fluids and fluids will also determine fluid distribution on the mesoscale.

Mesoscopic fluid distributions have been observed in recent experiments [35, 36, 60]. In these studies, clusters or patches of different pore fluids are distributed through out the porous rock samples. These experiments have revealed that the shape and distribution of mesoscopic fluid patches depends upon the degree of saturation and also upon the process of fluid saturation.

X-ray microtomographic images of Cadoret et al [36] show that imbibition experiments where water displaces gas, produce regular patches of fluids distributed uniformly 
through out the porous rock at high water saturations, whilst drainage or evaporative experiments, where the reverse fluid substitution process occurs, produce gas clusters distributed non-uniformly through out the porous rock at high water saturations.

As drainage and imbibition produce different saturation patterns at the same level of saturation, differences in attenuation and phase velocity measurements can be attributed to differences in fluid distribution. Moreover, phase velocities measured from drainage experiments are appreciably higher than those from imbibition experiments $[35,61]$ and similarly for attenuation measurements [36]. Thus, estimates of attenuation and phase velocity are affected by the distribution of immiscible fluids.

\subsection{Low and high frequency elastic moduli}

In response to a passing compressional wave, the porous framework of grains is compressed and rarefied on time scales imposed by the wave speed. When heterogeneities in saturating fluids (and or rock properties) exist, the compression or rarefaction of the frame causes spatial gradients in fluid pressure to develop. Providing that heterogeneities exist on length scales less than a wavelength, but greater than pore scale, gradients in fluid pressure develop on the mesoscale. This drives the so called mesoscopic fluid flow, which causes the attenuation of elastic energy and the dispersion of a propagating wave form. 
In the limiting cases of very low and very high wave frequencies, theoretical values of phase velocities can be determined. For intermediate wave frequencies, phase velocities are frequency dependent and lie between these limiting values. Following Mavko and Murkerji, [62], Johnson [8], Müller and Gurevich [11], the upper and lower-frequency limits on phase velocities are presented below.

For a porous rock having only heterogeneities in saturating fluids, Norris [63] has shown that the distribution of fluid pressures is governed by the diffusion equation with a diffusion length of $\lambda_{d}=\sqrt{\frac{\kappa N}{\omega \eta}}$,

where $N=M L / H, \omega$ is wave frequency, and $L$ and $H$ are $P$ wave moduli of the dry and fluid-saturated rock, respectively.

When the frequency $\omega$ of the incident wave is sufficiently low and the characteristic patch size of fluid heterogeneities is less then the diffusion length $\lambda_{d}$, there is enough time for fluid to flow and equilibrate at a constant pressure. In this limit, Wood's law [6] can be applied to determine an effective fluid bulk modulus $K_{f}$ given by,

$K_{W}=\left\lfloor S_{1} / K_{f 1}+S_{2} / K_{f 2}\right\rfloor$,

where $S_{1}, S_{2}$ are volume concentrations of fluids having bulk moduli $K_{f 1}, K_{f 2}$. 
Once the effective bulk modulus of the pore fluid is defined, Gassmann relations (7)-(9) can be applied to estimate the low-frequency phase velocity for a partially fluid saturated rock (Figure 3). This quasi-static limit is known as uniform saturation or GassmannWood limit.

Conversely, when the wave frequency $\omega$ is sufficiently high, and the characteristic patch size is larger than the diffusion length $\lambda_{d}$, there isn't enough time for pressure equilibration and fluid flow effects can be ignored. In this circumstance, patches of rock will remain at different pressures.

Application of Gassmann's theory on individual patches allows the saturated bulk modulus of each patch to be determined. According to Gassmann's equation (9), the saturated shear modulus of each patch is independent of fluid bulk modulus. Thus Hill's [64] theorem can be applied to determine the overall saturated bulk modulus:

$\left[K_{H}+4 \mu / 3\right]^{-1}=S_{1} /\left(K_{1}+4 \mu / 3\right)+S_{2} /\left(K_{2}+4 \mu / 3\right)$,

where $K_{1}$ and $K_{2}$ are the saturated bulk moduli determined by applying Gassmann's theory on each fluid patch (Figure 3). This high-frequency or no-flow limit is known as patchy or Gassmann-Hill limit. 
The elastic moduli in both the low- and high frequency limits are given by real numbers and are frequency independent. Johnson [8] has shown that (1) for any non-zero saturation the homogeneous moduli are always smaller than those for patchy saturation, and (2) at intermediate frequencies the bulk modulus lies between these limits. Thus for any intermediate saturation level the partially fluid saturated rock exhibits frequency dependent phase velocity.

\subsection{Regular cell models}

There are a number of approaches to modelling attenuation and phase velocity dispersion due to mesoscopic fluid flow. Most approaches assume that heterogeneities in fluid content or lithology, are distributed periodically throughout the porous medium. This approach was proposed by White et al [30] and White [7], who was the first to illustrate that significant amounts of attenuation and phase velocity dispersion could arise from mesoscopic fluid flow. White et al [30] modelled fluid heterogeneities as periodically alternating layers of gas and water in a uniform solid frame. White [7] modelled fluid heterogeneities as a periodic array of spherical gas inclusions, embedded within a watersaturated host (Figure 4).

In these approaches an elementary composite volume consisting of porous rock saturated by each fluid, is considered representative of the entire periodic system of fluid heterogeneities. In 1D, the representative volume spans the interface between different 
fluid layers from the centre of each layer. In 3D the representative volume is spherical enclosing a single gas inclusion. See figure 4.

In [30] and [7] the frequency dependent complex bulk modulus is derived by considering the ratio of the imposed pressure amplitude to the corresponding fractional change in volume (including effects of fluid flow). Later these models were recast using Biot's equations of dynamic poroelasticity [3] for 1D periodic layering by Norris [63] and for 3D spherical gas inclusions by Dutta and Ode [37,38]. These studies validated the conclusions that wave induced fluid flow causes attenuation and phase velocity dispersion.

Recently, two more general models for patchy saturation have been developed which also utilize 3D regular patch geometries [8,9]. These new models allow attenuation and phase velocity to be determined for arbitrary shaped fluid inclusions. However, explicit analytical expressions are only given for the concentric sphere geometry of White [7].

Johnson's APS (acoustics of patchy saturation) approach [8] was developed within the context of the low-frequency Biot theory. The dynamic bulk modulus $K(\omega)$ of a partially fluid saturated porous rock is developed by firstly considering its response to low and high wave frequencies. 
When wave frequencies are sufficiently low, the rock is "relaxed" as fluid pressure is equilibrated. In this limit the low frequency asymptote of $K(\omega)$ converges to GassmannWood limits (see section 2.2). Conversely, when wave frequencies are sufficiently high, the rock is "unrelaxed" as fluid pressures are unequilibrated. In this limit, the high frequency asymptote of $K(\omega)$ converges to Gassmann-Hill limit (see section 2.2). For intermediate frequencies, the dynamic response of the porous rock is constructed using a branching function, which ensures causality of the solution and convergence to higher and lower limits.

In the APS approach the dependency $K(\omega)$ is controlled by two new geometrically significant parameters: the specific surface area of the inclusion/composite volume and an effective patch size parameter. Utilizing this theory, Tserkovnyak and Johnson [65] deduced values for the specific surface area and effective patch size from experimental data $[35,36]$. They found that APS theory could be used to interpret geometrical measures of partial fluid saturation from attenuation and phase velocity measurements.

A more general approach based broadly on similar principles was recently developed by Pride and Berryman [66,67]. This approach yields estimates of attenuation and phase velocity in a general double-porosity dual-permeability medium. The theory utilizes Biot's equations [3] of poroelasticity to determine the poroelastic response of a composite body comprising of two distinct poroelastic phases. Pride et al [9] specialised general results of $[66,67]$ to the specific case of patchy saturation, where only heterogeneities in 
saturating fluids exist. The results for this special case are very similar to the results of Johnson [8].

Central to the double-porosity dual-permeability theory is a model for fluid transport between two poroelastic phases when induced fluid pressures are different. Fluid flow between phases is assumed to be proportional to the pressure difference, where the proportionality coefficient is frequency dependent. First, this fluid transport coefficient is determined for low and high frequencies. Then, following an approach of Pride et al [68], a branching function is introduced which links low and high frequency limits to determine the overall frequency dependence of the fluid transport coefficient.

All of the above approaches are limited to modelling attenuation and phase velocity dispersion due to wave induced flow arising between fluid heterogeneities which are identical and distributed regularly through out the porous medium. This is a consequence of decomposing the porous medium into identical cells (or composite volumes) containing fluid heterogeneities of regular shape. The use of idealised modelling geometries has clearly been helpful in identifying the effects of mesoscopic fluid flow. However, more realistic modelling geometries are required in order to evaluate how fluid distribution can firstly affect attenuation and phase velocity estimates and secondly to assist with the interpretation of experimental data. 


\section{Random patchy saturation models}

Attenuation and phase velocity dispersion due to a random distribution of poroelastic layers were first studied by Gurevich and Lopatnikov $[10,69,70]$ by analysing Biot's equations of poroelasticity with coefficients considered as random functions of one coordinate. The results of this analysis showed that random and periodic layering produces different attenuation behaviour at low frequencies. The frequency dependent attenuation for random layers was proportional to $\omega^{1 / 2}$, whilst for periodic layers, it was proportional to $\omega$. Müller and Gurevich [11] specialised those results to the case of patchy saturation, where only heterogeneities in fluid properties existed. Shapiro and Müller [71] showed how such models can be used in media with spatial fluctuations of permeability as well as fluid properties; see also [72 ].

The above results are applicable for layered porous media, that is to $1 \mathrm{D}$ distributions of heterogeneities in fluid properties and or rock properties. Hence, the question arises whether discrepancies in attenuation behaviour will also be manifested in 3D distributions of heterogeneities, which are periodic or random. This question, along with the general need for modelling more of realistic distributions of heterogeneities, has provided the impetus for the development of models based on the concepts of random media.

Two such approaches have recently been developed. The first approach models wave propagation in a porous medium with a random distribution of discrete inclusions of another porous medium $[12,73]$. The second approach considers waves in a porous 
medium whose properties are smooth functions of spatial coordinates $[74,75]$. In the following we review these two approaches and show how they can be used to estimate elastic wave attenuation and dispersion due to patchy saturation.

\subsection{Discrete random model}

Ciz et al $[12,73]$ have derived explicit expressions for attenuation and phase velocity dispersion due to a random distribution of spherical heterogeneities within porous rock (discrete random model or DRM). The derivation involves two main stages. In the first stage a problem of scattering by a single inclusion is analysed. Under the assumption of mesoscopic inclusion this analysis yields a closed-form solution for the scattering amplitude [76]. The second stage utilizes Waterman and Truell [77] theorem of multiple scattering to approximate the scattered wave field of a system of randomly distributed poroelastic inclusions [73].

\subsubsection{Single inclusion scattering}

The problem of scattering of an elastic wave in a poroelastic material (host) by a spherical inclusion of another poroelastic material (inclusion) was first considered by Berryman [78]. When the incident wave interacts with the inclusion of radius $a$, it produces fast and slow compressional waves and a shear wave in the host (called scattered or reflected waves) and three of the same kinds of waves within the inclusion (called refracted waves), Figure 6. 
Both the inclusion and host medium are described by Gassmann equations [4] and Biot's equations of poroelasticity (see section 1). Standard boundary conditions [79] apply on the interface between the inclusion and host at $r=a$ :

I. continuity of normal stress

II. continuity of tangential stress

III. continuity of normal average solid displacement

IV. continuity of tangential average solid displacement

V. continuity of fluid pressure

VI. continuity of average relative solid-fluid displacement

Similarly to the corresponding scattering problem in elasticity [80], the solution of poroelastic scattering problem is sought for by expanding the reflected and refracted waveforms in series of spherical harmonics [78]:

$u_{1 r}=-\sum_{n=0}^{\infty}\left[\frac{B_{n}^{+}}{k_{+}^{2}} \frac{d}{d r} h_{n}^{(1)}\left(k_{+} r\right)-\frac{B_{n}^{-}}{k_{-}^{2}} \frac{d}{d r} h_{n}^{(1)}\left(k_{-} r\right)\right] P_{n}(\cos \theta)$,

$u_{3 r}=\sum_{n=0}^{\infty}\left[\frac{D_{n}^{+}}{\left(k_{+}^{\prime}\right)^{2}} \frac{d}{d r} j_{n}\left(k_{+}^{\prime} r\right)-\frac{D_{n}^{-}}{\left(k_{-}^{\prime}\right)^{2}} \frac{d}{d r} j_{n}\left(k_{-}^{\prime} r\right)\right] P_{n}(\cos \theta)$,

where, $u_{1 r}$ and $u_{3 r}$ are normal displacements in the host and inclusion and $B_{n}^{+}, B_{n}^{-}$, $D_{n}^{+}, D_{n}^{-}$are coefficients corresponding to reflected and refracted fast and slow harmonics 
of order $n, j_{n}^{(1)}, h_{n}^{(1)}$, are spherical Bessel functions of the first and third kind, $P_{n}(\cos \theta)$ is the Legendre polynomial of the order $n$ and $k_{+}, k_{-}$are wave numbers of the fast and slow waves. Similar representation is derived for the polar angle components of displacements, which involve scattering coefficients $C_{n}$ and $E_{n}$ corresponding to reflected and refracted shear wave.

Application of the standard boundary conditions yields a $6 \times 6$ system of linear equations in terms of six unknown wave field coefficients of each order $n \geq 1$. For order $n=0$ a similar $4 \times 4$ system of linear equations in terms of 4 unknown wave field coefficients is obtained [78].

The general infinite series formulation of Berryman [78] gives a complete solution of the single scattering problem for an inclusion of arbitrary size (larger than the pore size) and for any frequency. Ciz and Gurevich [76] showed that this solution can be greatly simplified if the frequency is small compared to Biot's characteristic frequency, and the inclusion is mesoscopic. In particular, they showed that at most three first terms of the series are significant in this case, and gave explicit analytical expressions for these terms.

Furthermore, it was shown that the term with $n=2$ is proportional to the difference in shear modulus between the inclusion and the host medium. Thus in the specific case where the inclusion differs from the host medium by the fluid properties only, this term can be neglected and the scattering coefficient of the fast compressional wave is given by the sum of the zero-order and first-order terms with coefficients [12]: 


$$
\begin{aligned}
& B_{0}^{+}=\frac{i \xi_{+}^{3} A_{0}\left(K-K^{\prime}\right)}{3 H^{\prime \prime}}+i \xi_{+}^{3} A_{0} H\left(\frac{C}{H}-\frac{C^{\prime}}{H^{\prime \prime}}\right)^{2} /\left[N^{\prime} \frac{j_{0}\left(\xi_{-}^{\prime}\right)}{j_{1}\left(\xi_{-}^{\prime}\right)} \xi_{-}^{\prime}-N \frac{h_{0}^{(1)}\left(\xi_{-}\right)}{h_{1}^{(1)}\left(\xi_{-}\right)} \xi_{-}\right] \\
& B_{1}^{+}=\frac{A_{0} \xi_{+}^{3}}{3}\left(1-\frac{\rho^{\prime}}{\rho}\right) .
\end{aligned}
$$

In these expressions, $\xi_{ \pm}=k_{ \pm} a$, a prime (or no prime) above a parameter denotes within the inclusion (or host), whilst $\rho$ refers to the effective density of the porous rock.

\subsubsection{Random distribution of inclusions}

Waterman and Truell [77] showed that the velocity and attenuation of waves propagating in a medium containing a random distribution of identical inclusions can be related to the amplitude of the waves scattered from a single inclusion (Figure 7). According to their theory, the complex effective wave number is given by:

$$
\left(\frac{k_{e f f}}{k_{+}}\right)^{2}=\left[1+\frac{2 \pi v f(0)}{k_{+}^{2}}\right]^{2}-\left[\frac{2 \pi v f(\pi)}{k_{+}^{2}}\right]^{2},
$$

where $k_{+}=\omega / v_{+}$is the wave number of the fast $P$-wave in the host, $v$ is the density or number of scatterers per unit volume, and $f(0), \quad f(\pi)$ are forward and backward scattering amplitudes which are related to scattering coefficients by

$$
f(0)=\frac{1}{i k_{+}} \sum_{n=0}^{\infty} i^{n} B_{n}^{+}, \quad f(\pi)=\frac{1}{i k_{+}} \sum_{n=0}^{\infty}(-i)^{n} B_{n}^{+},
$$


For random distribution of mesoscopic fluid patches the scattering amplitudes are determined by substituting scattering coefficients (13) and (14) into (16).

Incorporating a weak scattering approximation and neglecting quadratic terms in $v$ reduces the effective wave number (15) to

$k_{\text {eff }}=k_{+}\left[1+\frac{4 \pi v f(0)}{k_{+}^{2}}\right]^{1 / 2} \approx k_{+}\left[1+\frac{2 \pi v f(0)}{k_{+}^{2}}\right]$.

Real and imaginary components of $k_{\text {eff }}$, yield the effective phase velocity $v_{\text {eff }}$ and dimensionless attenuation (inverse quality factor) $Q^{-1}$

$$
\begin{aligned}
& \frac{1}{v_{e f f}}=\frac{1}{v_{+}}\left[1+\frac{3}{2} \frac{\delta}{\xi_{+}^{3}} \operatorname{Re}\left\{-i B_{0}^{+}+B_{1}^{+}\right\}\right], \\
& Q^{-1}=3 \delta \operatorname{Im}\left\{\frac{A_{0}\left(C H^{\prime \prime}-C^{\prime} H\right)^{2}}{H H^{\prime 2}\left[N^{\prime} \frac{j_{0}\left(\xi_{-}^{\prime}\right)}{j_{1}\left(\xi_{-}^{\prime}\right)} \xi_{-}^{\prime}-N \frac{h_{0}^{(1)}\left(\xi_{-}\right)}{h_{1}^{(1)}\left(\xi_{-}\right)} \xi_{-}\right]}\right\},
\end{aligned}
$$

where $\delta=v /\left(4 / 3 \pi a^{3}\right)$ is the fractional volume concentration of the inclusions.

The discrete random model discussed here is limited to the spherical shape of the patches, and in this respect is similar to regular patch models [7-9]. Unlike these models however, DRM is based on the scattering theory, and thus implies random distribution of the fluid patches. Another advantage of the DRM is it that gives explicit closed-form expressions for the frequency dependency of attenuation and dispersion. On the other hand, since the phase velocity and attenuation estimates (18) and (19) are based on an essentially single 
scattering approximation of Waterman and Truell, they are limited to small concentrations of inclusions. The range of admissible concentrations depends on the contrasts in fluid properties between the inclusion and the host medium. Numerical comparison of the DRM with other models will be presented in section 4.

\subsection{Continuous random model}

All the partial saturation models described so far assume a fixed shape (and size) of fluid patches. In real rocks however we expect fluid patches (bubbles) to have a broad distribution of shapes and sizes. Since effect of small patches can be adequately described by the Gassmann-Wood homogeneous saturation model (7)-(10), see [81], we can consider the partially saturated medium as a homogenous porous frame saturated with a composite fluid with smoothly varying properties. Such a medium can be described by Biot's equations of poroelasticity with poroelastic coefficients which are continuous random functions of position. This approach was first used to model wave propagation in randomly layered poroelastic media $[10,11]$. This was done using the so-called method of statistical smoothing [82], widely used in the theory of waves in random media. Recently Müller and Gurevich [74,75] used a similar approach to develop a general model of wave attenuation and dispersion in 3D randomly inhomogeneous porous media. The method of statistical smoothing is precise for small contrasts in physical properties.

According to Müller and Gurevich [74,75], the complex $P$-wave number in a heterogeneous porous solid is given by 


$$
\bar{k}_{e f f}=k_{+}\left(1+\Delta_{2}+\Delta_{1} k_{-}^{2} \int_{0}^{\infty} r B(r) \exp \left(i k_{-} r\right) d r\right)
$$

with dimensionless coefficients given by,

$$
\Delta_{1}=\frac{\alpha^{2} M L}{2 H^{2}}\left(\sigma_{L L}^{2}-2 \sigma_{L M}^{2}+\sigma_{M M}^{2}\right), \Delta_{2}=\frac{L}{2 H} \sigma_{L L}^{2}+\frac{\alpha^{2} M}{2 H} \sigma_{M M}^{2},
$$

where $k_{+}=\omega \sqrt{\rho / H}, k_{-}=\sqrt{i \omega \eta / \kappa N}$, are the fast and slow $P$-wave numbers for the background medium, $B(r)$ is the spatial correlation function and $\sigma_{x x}$ are normalised variances of the different elastic moduli.

For partial fluid saturation, only variances in fluid modulus $M$ exist, so that $\sigma_{L L}=\sigma_{L M}=0, \Delta_{2}=\alpha^{2} M \sigma_{M M}^{2} / 2 H$ and $\Delta_{1}=(L / H) \Delta_{2}$. This reduces the complexity of the dimensionless coefficients in (21). To obtain the fast and slow $P$ wave numbers for the average background media, it is necessary to calculate the average properties of the fluid modulus $M$, and fluid viscosity $\eta$. This is done by taking the saturation-weighted average of each property: $\langle X\rangle=X_{1} S_{1}+X_{2} S_{2}$.

Using (20), the effective complex $P$-wave modulus can be written as,

$$
H_{e f f}(\omega)=H_{0}\left(1-\Delta_{2}-\Delta_{1} k_{-}^{2} \int_{0}^{\infty} r B(r) \exp \left(i k_{-} r\right) d r\right)^{2}
$$

where $H_{0}$ is the average background $P$-wave modulus determined from Gassmann's equation using the average fluid modulus $M$. Real and imaginary components of (23) yield the effective phase velocity $v_{\text {eff }}=\sqrt{\operatorname{Re}\left\{H_{\text {eff }}\right\} / \rho}$ and specific attenuation (inverse quality factor) $Q_{e f f}^{-1}=\operatorname{Im}\left\{H_{e f f}\right\} / \operatorname{Re}\left\{H_{e f f}\right\}$ respectively. 
The theoretical low- and high-frequency limits on $P$ wave moduli obtained from (22) are $H_{\text {low }}=H_{0}\left(1-\Delta_{2}\right)^{2}$ and $H_{\text {high }}=H_{0}\left(1-\Delta_{2}+\Delta_{1}\right)^{2}$, respectively. For small contrast in fluid properties $\sigma_{M M} \ll<1$ these moduli approach theoretical Gassmann-Wood $H_{W}$ and Gassmann-Hill $H_{H}$ bounds as given by equations (6)-(10) and (11) respectively: $H_{\text {low }}=H_{W}+o\left(\sigma_{M M}\right)$ and $H_{\text {high }}=H_{H}+o\left(\sigma_{M M}\right)$. In other words, the moduli given by equation (22) are asymptotically consistent with Gassmann-Wood and Gassmann-Hill limits for small contrast in fluid modulus. However, in partially saturated rocks the variation in fluid properties is often large, resulting in significant deviation of predicted low- and high-frequency moduli from the theoretical limits.

To make the model consistent with the theoretical limits, we introduce a scaling function [11]:

$$
H_{S C}(\omega)=H_{W}\left(1+\frac{H_{H}-H_{W}}{H_{\text {high }}-H_{\text {low }}} \times \frac{H_{\text {eff }}(\omega)-H_{\text {low }}}{H_{W}}\right) .
$$

The new complex modulus $H_{S C}(\omega)$ predicted by equation (23) behaves similarly to that predicted by (22) but is consistent with the theoretical limits. We call this scaled model the continuous random model (CRM) of patchy saturation.

Dispersion and attenuation in CRM depends on the correlation function $B(r)$, which in turn is determined by the spatial distribution of saturating fluids. To obtain closed-form expressions one needs to specify this correlation function. In particular, for an 
exponential correlation function $B(r)=\exp (-|r| / b)$ the effective complex $P$-wave modulus in this case becomes

$$
H_{\text {eff }}(\omega)=H_{0}\left(1-\Delta_{2}-\frac{\Delta_{1} k_{-}^{2} b^{2}}{\left(i k_{-} b-1\right)^{2}}\right)^{2},
$$

where $b$ is the correlation length, which characterizes a characteristic length of the inhomogeneities.

To compare dispersion and attenuation estimates obtained from the random continuous distribution of fluids and discrete inclusion based methods, it is necessary to relate fundamental qualities which characterize each type of media. Random media is characterized by variance, correlation function and correlation length, whilst discrete media is characterized by inclusion radius, volume concentration and specific area. We have derived an approximate relationship by equating the power expansion of the exponential correlation function to the correlation function defined by Torquato [83] for a random distribution of non-overlapping spherical inclusions. By doing this we have obtained the relationship $b=4 S_{1} S_{2} / s_{v}$, which allows the correlation length $b$ to be computed from fluid saturations $S_{1}, S_{2}$ and specific volume $s_{v}=3 r_{i}^{2} / R_{c}^{3}$, where $r_{i}, R_{c}$ are inclusion and composite volume radius, respectively.

\section{Numerical examples}

The numerical examples below illustrate attenuation and phase velocity dispersion predicted using several different 3D modelling approaches. In particular, the periodic 
models of White, [7]; Johnson [8] and Pride and Berryman [9] will be compared with the random models. Typical physical properties we use in the following examples are given in Table 1.

Earlier approaches to modelling attenuation due to partial fluid saturation utilized Biot's equations of poroelasticity with an effective fluid bulk modulus defined by Wood's law [31]. For $15 \%$ air inclusions within an otherwise water saturated host rock of porosity 0.08 , estimates of attenuation are obtained using White's concentric sphere model and Biot-Wood theory (BW). Figure 8 shows that the peak magnitude of attenuation for BW theory is several orders of magnitude less than that predicted by White's model [7]. In particular, the peak frequency for BW theory is in the higher frequency bandwidths, whilst White's model is in the low (seismic) frequency bandwidth.

The models predict different magnitudes of attenuation at different frequencies as the dominating attenuation mechanisms are physically different. In White's model attenuation is a consequence of wave induced fluid flow occurring between the inclusion and composite host volume. This arises as the inclusion and host develop different fluid pressures. Where as, in BW theory it is assumed that both porefluids are equilibrated at a common fluid pressure, and thus fluid flow only occurs between the troughs and peaks of the incident wave.

The next example models attenuation and phase velocity using the different periodic models of White [7], Johnson [8] and Pride et al [9]. For 5\% of air inclusions within an 
otherwise water saturated host rock of porosity 0.15 , attenuation and phase velocity are shown in Figure 9 and 10, respectively. The figures show that there is a good agreement between all periodic models for estimates of attenuation and phase velocity. In particular, Figure 9 shows that attenuation at low frequencies is proportional to $\omega$, whilst for high frequencies it is proportional to $\omega^{-1 / 2}$. Figure 10, shows that phase velocities converge to low (Gassmann-Wood) and high frequency (Gassmann-Hill) limits and at intermediate frequencies the phase velocities are bounded by those limits. The periodic models [7-9] estimates of attenuation and dispersion are all in good agreement because they all share the same geometrical arrangement and distribution of the same pore fluids. Firstly, attenuation will be similar because it is dominated by concentration and contrast between the pore fluids (which is the assumed the same in this example). Secondly, they all share the same effective patch size, thus relaxation/unrelaxation will occur at similar frequencies.

The next example models attenuation and phase velocity due to a random distribution of spherical inclusions (using the DRM model above) and a periodic distribution of spherical inclusions (using White's model [7] as the reference model). In Figure 11, attenuation and phase velocity are shown when there are small contrasts in saturating fluids (a) $0.1 \%$ heavy gas and (b) $10 \%$ heavy gas within an otherwise water saturated rock. In both cases, the more compressible fluids are modelled as inclusions. In (c) the more compressible fluid is modelled as the host saturating fluid for the situation of large contrasts between fluids. Figure 11, top row (a) shows good agreement between attenuation and phase velocity estimates for small contrasts in fluid properties when the 
volume concentration of the included phase is small. Figure 11, middle row (b) shows a larger volume concentration of the included fluid results in different attenuation and phase velocity estimates. In particular, the phase velocity estimate of the DRM does not converge to the low frequency Gassmann-Wood limit. This is a consequence of the weak scattering approximation employed in the models derivation.

When the more compressible fluid is modelled as the host saturating fluid, the DRM can handle larger contrasts in fluid properties. In (c), attenuation and phase velocity dispersion is modelled for $50 \%$ water inclusions within an otherwise air saturated host rock of porosity 0.08 . There is very good agreement between attenuation and phase velocity estimates. Figure11, shows that the attenuation behaviour of periodic and random distributions of fluid inclusions is proportional to $\omega$ for low frequencies and proportional to $\omega^{-1 / 2}$ for high frequencies. Providing the weak scattering conditions are met, there is good agreement between attenuation and phase velocity estimates for periodic and random distributions of fluid inclusions.

The next example models attenuation and phase velocity using the continuous random model (CRM) and White's model. When comparing CRM to inclusion based models, it is necessary to formulate some type of relationship which allows significant parameters that characterise each approach to assume equivalent measures. We assume that specific surface area can be used as a means of relating the two formulations and that the continuous random distribution of fluids is described by an exponential correlation 
function. In Figure 12 two types of models are compared with a water saturated host rock, (a) when there are $35 \%$ of air inclusions and (b) when there are $35 \%$ of heavy gas inclusions. Figure 12, shows that the CRM estimates attenuation magnitudes which are of the same magnitude as the periodic model and that attenuation behaviour has the same frequency behaviour at low and high frequencies. The attenuation peak is broader for the CRM model and the bandwidth over which phase velocity dispersion occurs is larger for the CRM model. This is expected as CRM implicitly assumes that there is a range of patch sizes and thus larger patch sizes will become unrelaxed at lower frequencies than smaller patch sizes. Figure 12 also shows that the CRM model can handle large and small contrasts in fluid properties equally well.

Figure 13, shows attenuation estimates obtained using the 3D and 1D variations of CRM with the periodic reference model of White [7]. These estimates are compared with experimental measurements obtained from Murphy [84]. The figure shows that the 3D and 1D variations of CRM produce the closest estimates of attenuation. The reason why the 1D random model explains the data best is that in the experimental setup of Murphy the fluid distribution is likely to be given by a realization of a strongly anisotropic (quasi1D) random medium. That is, the pore fluid distribution is likely to have greater variance in $1 \mathrm{D}$ then across 3D. 


\section{Conclusions}

The partial saturation of a porous rock by different types of fluids can cause the attenuation and dispersion of seismic waves. This effect is a consequence of waveinduced flow, which arises when a passing wave induces different fluid pressures in regions of rock saturated by different fluid types. The presence of localized spatial gradients in fluid pressure causes fluid to flow and the viscous dissipation of energy. Depending on the size of fluid clusters these gradients may occur on the pore scale or on the mesoscale (a scale that is larger than the pore size but smaller than wavelength scale). Pore-scale fluid heterogeneity causes local flow, while dissipation caused by mesoscale fluid distribution is called mesoscopic.

The most common approach to modelling mesoscopic attenuation and dispersion is based on the assumption that the medium can be divided into identical cells containing regularly shaped fluid patches of the two fluids. 1D heterogeneities are modelled using periodically alternating layers, whilst in 3D an array of spherical inclusions are positioned at regular lattice positions.

Real distributions of fluid in the pore space of the rock are unlikely to form regular patterns. An alternative approach is to consider a random distribution of fluids. For the 1D case (horizontally layered medium) this was done by assuming that fluid properties are random functions of one spatial coordinate, and by analysing the solution of Biot's equations of poroelasticity with random coefficients. This analysis shows that frequency 
dependence of attenuation caused by randomly stratified fluid distribution is very different from that caused by periodically layered distribution. In particular, at low frequencies inverse $Q$ is proportional to $\omega^{1 / 2}$ for random layering, whilst to $\omega$ for periodic layering.

Recently, two models have been proposed that attempt to simulate three-dimensional random distributions of fluids. The first model assumes that one fluid forms a random ensemble of spherical inclusions in a porous medium saturated by the other fluid. However, despite the fact that the distribution of fluid patches is random, the resulting attenuation and dispersion are essentially the same as for a periodic distribution of spherical fluid clusters. However this agreement may be the result of the limitation of the random model to low concentration of inclusions, where inclusion interactions are negligible.

Another model proposed by the present authors considers the fluid properties as continuous random functions of position in 3D. This approach, which can be considered as an extension of the 1D random model to three dimensions, leads to frequency dependency of attenuation whose low and high frequency asymptotes are the same as in models with periodic patches, but whose shape is significantly different. This difference is the consequence of the fact that the continuous random model implies a broad range of fluid patches, whereas models with fixed-shaped inclusions (periodic or random) the fluid patch size (as well as shape) is fixed. 
Comparisons with experimental data appear to suggest that the continuous random model is more adequate than models with regular distribution of fluid patches. However, further research is required to relate the parameters of natural fluid distributions (both in the

laboratory and field experiments) to the parameters of random functions used in the model.

\section{Acknowledgements}

This work was kindly supported by the Centre of Excellence for Exploration and Production Geophysics, Curtin Reservoir Geophysics Consortium, CSIRO + WACUPS postgraduate scholarships, CSIRO Postdoctoral Fellowship Program, and CSIRO Petroleum and Deutsche Forschungsgemeinschaft (contract MU 1725/1-1). 


\section{Appendix: Symbols and terms}

$a$ inclusion radius

$b$ correlation length

$A_{0}$ amplitude of incident wave

$B(r)$ correlation function

$B_{n}^{+}, B_{n}^{-}$fast and slow wave reflected scattering coefficient

$C_{n}$ reflected shear wave scattering coefficient of order $\mathrm{n}$

$D_{n}^{+}, D_{n}^{-}$fast and slow wave refracted scattering coefficient

$E_{n}$ refracted shear wave scattering coefficient of order $\mathrm{n}$

$f(0), f(\pi)$ forward and backward scattering amplitudes eq(16)

$f_{c}$ Biot characteristic frequency

$h_{n}^{(1)}$ spherical Bessel function of the third kind of order $\mathrm{n}$

$H$ saturated $\mathrm{P}$ wave modulus

$H_{0}$ average background medium saturated $\mathrm{P}$ wave modulus

$H_{H}$ saturated $\mathrm{P}$ wave modulus defined by Gassmann-Hill limit

$H_{W}$ saturated $\mathrm{P}$ wave modulus defined by Gassmann-Wood limit

$H_{\text {eff }}$ effective medium saturated $\mathrm{P}$ wave modulus eq (22), eq(24)

$H_{\text {low }}$ low frequency limit of effective $\mathrm{P}$ wave modulus

$H_{\text {high }}$ high frequency limit of effective $\mathrm{P}$ wave modulus

$H_{S C}$ scaled $\mathrm{P}$ wave modulus eq (23)

$i \sqrt{-1}$

$j_{n}^{(1)}$ spherical Bessel function of the first kind of order $\mathrm{n}$

$k_{\text {eff }}$ effective wave number eq(15), eq (17)

$\bar{k}_{\text {eff }}$ effective wave number eq (20)

$k_{+}, k_{-}$fast and slow $\mathrm{P}$ wave numbers eq(6), eq(

$K$ fluid saturated eq (7)

$K_{1}, K_{2}$ bulk modulus of patch saturated with fluid type 1,2

$K_{d}$ dry rock frame bulk modulus

$K_{f}, K_{f 1}, K_{f 2}$ fluid bulk modulus

$K_{g}$ grain bulk modulus

$K_{H}$ bulk modulus derived from Hill's theorem eq(11)

$K_{W}$ bulk modulus derived from Wood's theorem eq (10)

$L$ dry $\mathrm{P}$ wave modulus

$M$ fluid modulus (8)

$P_{n}(\cos \theta)$ Legendre polynomial of order $\mathrm{n}$

$q$ coupling coefficient between fluid and solid eq(3), eq(4) 
$Q_{+}^{-1}, Q_{-}^{-1}$ attenuation factors fast and slow $\mathrm{P}$ waves

$r_{i} R_{c}$ radius of inclusion and composite volume

$s_{v}$ specific area of fluid patches

$S_{1}, S_{2}$ volume concentration of fluid type1 and type 2

$\mathbf{u}$ average solid displacement

$u_{r 1}, u_{r 3}$ normal displacements in the host and inclusion eq (12)

$\mathbf{U}$ average fluid displacement

$v_{+}, v_{-}$fast and slow $\mathrm{P}$-wave velocities

$v_{\text {eff }}$ effective $\mathrm{P}$ wave velocity

$\mathbf{w}$ average relative fluid displacement

\section{Greek letters}

$\alpha$ Biot Willis coefficient

$\delta$ fractional volume concentration of inclusions

$\Delta_{1}, \Delta_{2}$ dimensionless coefficients eq(21)

$\xi_{+}$fast wavenumber (host) $\times$inclusion radius

$\xi$ slow wavenumber (host) $\times$ inclusion radius

$\xi_{+}^{\prime}$ fast wavenumber (inside inclusion) $\times$ inclusion radius

$\xi_{-}$slow wavenumber (inside inclusion) $\times$inclusion radius

$\eta$ fluid viscosity

$\kappa$ permeability

$\kappa^{*}$ dynamic hydraulic permeability

$\lambda_{d}$ diffusion length

$\mu$ saturated shear modulus eq(9)

$\mu_{0}$ unsaturated shear modulus

$\mu_{g}$ grain shear modulus

$v$ is the density or number of scatterers per unit volume

$\rho$ effective density of fluid saturated rock

$\rho_{0}$ rock frame density

$\rho_{f}$ fluid density

$\rho_{g}$ rock grains density

$\sigma_{L L}^{2} \sigma_{M M}^{2}$ variance of dry $\mathrm{P}$ wave modulus and fluid modulus

$\sigma_{L M}^{2}$ cross-variance of dry $\mathrm{P}$ wave modulus and fluid modulus

$\phi$ porosity

$\chi$ tortuosity

$\omega$ angular frequency 


\section{References}

[1] Biot MA. Theory of propagation of elastic waves in a fluid in a fluid saturated porous solid. .I.-Low frequency range. J Acoust Soc Am 1956a; 28: 168-178

[2] Biot MA. Theory of propagation of elastic waves in a fluid saturated porous solid. II. Higher frequency range. J Acoust Soc Am 1956b; 28: 179-191

[3] Biot MA. Mechanics of deformation and acoustic propagation in porous media. J Appl Phys 1962; 33: 1482-1498

[4] Gassmann F. Elastic waves through a packing of spheres. Geophysics 1951; 16: 673685.

[5] Bear J. Dynamics of fluids in porous media. New York: Dover; 1988

[6] Wood AB. A textbook of sound. London: Bell; 1941

[7] White JE. Computed seismic speeds and attenuation in rocks with partial gas saturation. Geophysics 1975; 40: 224-232

[8] Johnson DL. Theory of frequency dependent acoustics in patchy-saturated porous media. J Acoust Soc Am 2001; 110: 682- 694

[9] Pride SR, Berryman JG, Harris JM. Seismic attenuation due to wave-induced flow. J Geophys Res 2004; 109: No. B1, B01201

[10] Gurevich B, Lopatnikov SL. Velocity and attenuation of elastic waves in finely layered porous rocks. Geophys J Int 1995; 121: 933-947.

[11] Müller TM, Gurevich B. One-dimensional random patchy saturation model for velocity and attenuation in porous rocks. Geophysics 2004; 69: 1166-1172.

[12] Ciz R, Toms J, Gurevich B. Attenuation and dispersion of elastic waves in a poroelastic medium with spherical inclusions, $3^{\text {rd }}$ Biot Conference on Poromechanics, Oklahoma, in print; 2005

[13] Toms, J., Müller, T., and Gurevich, B., (2005), 3D random patchy saturation model for velocity and attenuation in porous rock, 75th Ann. Internat. Mtg.: Soc. of Expl. Geophys. 2005, Houston, Expanded Abstracts, paper SM 1.1: 1747-1750.

[14] Pride SR, Gangi AF, Morgan FD. Deriving the equations of motion for porous isotropic media. J Acoust Soc Am 1992; 92: 3278-3290 
[15] Levy T. Propagation of waves in a fluid-saturated porous elastic solid. Int J Engng Sci 1979; 17: 1005-1014

[16] Auriault JL. Dynamic behaviour of a porous medium saturated by a newtonian fluid. Int J Engng Sci 1980; 18: 775-785.

[17] Burridge R, Keller JB. Poroelasticity equations derived from microstructure. J Acoust Soc Am 1981; 70: 1140-1145

[18] Biot MA, Willis DG. The elastic coefficients of the theory of consolidation. J App Mech 1957; 24: 594-601

[19] Bourbie T, Coussy O, Zinszner B. Acoustics of Porous Media. Paris: Technip; 1987.

[20] Berryman JG. Seismic wave attenuation in fluid saturated porous media. PAGEOPHY 1998; 128: 424-432

[21] Wang Z. Fundamentals of seismic rock physics. Geophysics 2001; 66: 398-412

[22] Smith TM, Sondergeld CH, Rai CS. Gassmann fluid substitutions: A tutorial. Geophysics 2003; 68: 430-440

[23] Johnston DH, Toksöz, MN, Timur A. Attenuation of seismic waves in dry and saturated rocks: Mechanisms. Geophysics 1979; 44: 691-711

[24] Winkler K. Dispersion analysis of velocity and attenuation in Berea sandstone. J Geophys Res 1985; 90: 6793-6800

[25] Gist GA. Interpreting laboratory velocity measurements in partially gas-saturated rocks. Geophysics 1994; 59: 1100-1109

[26] Buckingham MJ. Wave propagation, stress relaxation, and grain-to-grain shearing in saturated, unconsolidated marine sediments. J Acoust Soc Am 2000; 108: 2796-2815

[27] Plona TJ. Observation of a second bulk compressional wave in a porous medium at ultrasonic frequencies. Appl Phys Lett 1980; 36: 259-261

[28] Nagy PB, Alder L, Bonner BP. Slow wave propagation in air-filled porous materials and natural rocks. Appl Phys Lett 1990; 56: 2504-2506

[29] Kelder O, Smeulders DMJ. Observation of the Biot slow wave in water-saturated Nivelsteiner sandstone. Geophysics 1997; 62: 1794-1796

[30] White JE, Mikhaylova NG, Lyakhovitskiy FM. Low-frequency seismic waves in fluid-saturated layered rocks. Phys Solid Earth Trans Izv 1976; 11: 654-659 
[31] Domenico SN. Effect of brine-gas mixture on velocity in an unconsolidated sand reservoir. Geophysics 1976; 41: 882-894

[32] Gregory AR. Fluid saturation effects on dynamic elastic properties of sedimentary rocks. Geophysics 1976; 41: 895-921

[33] Murphy WF. Acoustic measures of partial gas saturation in tight sandstones. Journal of Geophysical Research 1984; 89 B13: 11549-11559

[34] Murphy WF. Sonic and ultrasonic velocities: theory versus experiment. Geophysical Research Letters 1985; 12: 85-88

[35] Cadoret T, Marion D, Zinszner B. Influence of frequency and fluid distribution on elastic wave velocities in partially saturated limestones. J Geophys Res 1995; 100: 97899803

[36] Cadoret T, Mavko G, Zinszner B. Fluid distribution effect on sonic attenuation in partially saturated limestones. Geophysics 1998; 63: 154-160.

[37] Dutta NC, Ode H. Attenuation and dispersion of compressional waves in fluid-filled porous rocks with partial gas saturation (White model)- Part I. Biot theory. Geophysics 1979a; 44: 1777-1788

[38] Dutta NC, Ode H. Attenuation and dispersion of compressional waves in fluid-filled porous rocks with partial gas saturation (White model)-Part II. Results. Geophysics $1979 b ; 44: 1789-1805$

[39] Dutta NC, Seriff AJ. On White's model of attenuation in rocks with partial gas saturation. Geophysics 1979; 44: 1806-1812

[40] Carcione JM, Helle HB, Pham NH. White's model for wave propagation in partially saturated rocks, Comparison with poroelastic numerical experiments. Geophysics 2003; 67: 1389-1398.

[41] Helle HB, Pham NH, Carcione JM. Velocity and attenuation in partially saturated rock, poroelastic numerical experiments. Geophysical Prospecting 2003; 51: 551-566

[42] Mavko G, Nur A. Wave propagation in partially saturated rocks. Geophysics 1979; 44:161-178

[43] Palmer ID, Traviola ML. Attenuation by squirt flow in undersaturated gas sands. Geophysics 1980; 45: 1780-1792

[44] Murphy WA, Winkler KW, Kleinberg RA. Acoustic relaxation in sedimentary rocks- Dependence on grain contacts and fluid saturation. Geophysics 1986; 51: 757-766 
[45] Jones TD. Pore fluids and frequency-dependent wave propagation in rocks. Geophysics 1986; 51: 1939-1953

[46] Bedford A, Stern M. A model for wave propagation in gassy sediments. J Acoust Soc Am 1982; 73: 409-417

[47] Lopatnikov SL, Gorbachev PY. Propagation and attenuation of longitudinal waves in a partially gas-saturated porous media. Izvestiya Earth Physics 1987; 23: 683-689

[48] Smeulders DMJ, van Dongen MEH. Wave propagation in porous media containing a dilute gas-liquid mixture: theory and experiments. J Fluid Mech 1997; 343: 351-373

[49] Auriault JL, Boutin C, Royer P, Schmitt D. Acoustics of a porous medium saturated by a bubbly fluid undergoing phase change. Transport in porous media 2002; 46: 43-76

[50] Assefa S, McCann C, Sothcott J. Attenuation of P- and S-waves in limestones. Geophys Prosp 1999; 47: 359-392

[51] Best AI, McCann C, Sothcott J. The relationship between the velocities, attenuations and petrophysical properties of reservoir sedimentary rocks. Geophys Prosp 1994; 42: $151-178$

[52] Silberman EJ. Sound velocity and attenuation in bubbly mixtures measured in standing wave tubes. J Acoust Soc Am 1957; 29: 925-933

[53] van Wijngaarden L. One dimensional flow of liquids containing small gas bubbles. Ann Rev Fluid Mech 1972; 4: 369-396

[54] Commander KW, Prosperetti A. Linear pressure waves in bubbly fluids. Comparison between theory and experiments. J Acoust Soc Am 1989; 85: 732-746

[55] Knight R, Chapman A, Knoll M. Numerical modelling of microscopic fluid distribution in porous media. J Appl Phys 1990; 68: 994-1001

[56] Silverstein DL, Fort T. Prediction of air-water interfacial air in wet unsaturated porous media. Langmuir 2000a; 16: 829-834

[57] Silverstein DL, Fort T. Incorporating low hydraulic conductivity in a numerical model for predicting air-water interfacial area in wet unsaturated particulate porous media. Langmuir 2000b; 16: 835-838

[58] Silverstein DL, Fort T. Prediction of water configuration in wet unsaturated porous media. Langmuir 2000c; 16: 839-844

[59] Berkowitz B, Hansen D. A numerical study of the distribution of water in partially saturated porous rock. Transport in Porous Media 2001; 45: 303-319 
[60] Monsen K, Johnstad SE. Improved understanding of velocity-saturation relationships using 4D computer-tomography acoustic measurements: Geophys Prosp 2005; 53: 173-181

[61] Knight R, Nolen-Hoeskema R. A laboratory study of the dependence of elastic wave propagation of elastic wave velocities on porescale distribution. Geophys Res Lett 1990; $17: 1529-1532$

[62] Mavko G, Mukerji T. Bounds on low frequency seismic velocities in partially saturated rocks. Geophysics 1998; 63: 918-924

[63] Norris AN. Low-frequency dispersion and attenuation in partially saturated rocks. J Acoust Soc Am 1993; 94: 359-370

[64] Hill R. Elastic properties of reinforced solids: some theoretical principles. J Mech Phys Solids 1963; 11: 357-372

[65] Tserkovnyak Y, Johnson DL. Can one hear the shape of a saturation patch. Geophys Res Lett 2002; 29: 1108-1112

[66] Pride SR, Berryman JG. Linear dynamics of double-porosity and dual-permeability materials. I. Governing equations and acoustic attenuation. Phys. Rev. E 2003a; 68: 036,603,

[67] Pride SR, Berryman JG. Linear dynamics of double-porosity and dual permeability materials. II. Fluid transport equations. Phys. Rev. E 2003b; 68: 036,604

[68] Pride SR, Morgan FD, Gangi AF. Drag forces of porous-medium acoustics. Phys. Review B 1993; 47: 4964-4978

[69] Lopatnikov SL, Gurevich B. Attenuation of elastic waves in a randomly inhomogeneous saturated porous medium, Doklady Earth Science Sections 1986; 291(6): $19-22$.

[70] Gelinsky S, Shapiro SA, Müller TM, Gurevich B. Dynamic poroelasticity of thinly layered structures. International Journal of Solids and Structures 1998; 35: 4739-4752

[71] Shapiro SA, Müller TM. Seismic signatures of permeability in heterogeneous porous media. Geophysics 1999; 64: 99-103

[72] Müller TM, Lambert G, Gurevich B. Dynamic permeability of random porous rocks and its seismic signatures. $75^{\text {th }}$ Ann. Internat. Mtg.: Soc. of Expl. Geophys. 2005, Houston, Expanded Abstracts, paper RP 1.8: 1497-1500. 
[73] Ciz R, Gurevich B., Seismic attenuation in a porous rock with spherical heterogeneities, 74rd Ann. Internat. Mtg.: Soc. of Expl. Geophys. 2004, Denver, Expanded Abstracts, paper 466.

[74] Müller TM, Gurevich B. A first order statistical smoothing approximation for the coherent wave field in random porous media. J Acoust Soc Am 2005a; 117: 1796-1805

[75] Müller TM, Gurevich B. Wave induced fluid flow in random porous media: Attenuation and dispersion of elastic waves. J Acoust Soc Am 2005b; 117: 2732-2741

[76] Ciz R, Gurevich B. Amplitude of Biot's slow wave scattered by a spherical inclusion in a fluid-saturated poroelastic medium. Geophys J Int 2005; 160: 991-1005

[77] Waterman PC, Truell R. Multiple scattering of waves. J Math Phys 1961; 2 (4): 512 $-537$.

[78] Berryman JG Scattering by a spherical inhomogeneity in a fluid-saturated porous medium. J Math Phys 1985; 26 (6): 1408-1419.

[79] Deresiewicz H, Skalak R. On uniqueness in dynamic poroelasticity. Bull Seis Soc Am 1963; 53: 409-416.

[80] Yamakawa N. Scattering and attenuation of elastic waves. Geophys. Mag., 31, 6397, Tokyo, 1962

[81] Knight R, Dvorkin J, Nur A. Acoustic signatures of partial saturation. Geophysics $1998 ; 63: 132-138$

[82] Karal FC, Keller JB. Elastic, electromagnetic and other waves in random. J Math Phys 1964; 5: 537-547

[83] Torquato S. Random Heterogeneous Materials: Microstructure and Macroscopic Properties. New York: Springer-Verlag; 2001

[84] Murphy WF. Effects of partial water saturation on attenuation in Massilon sandstone and Vycor porous glass. J Acoust Soc Am 1982; 71: 1458-1468 


\section{Figure Captions:}

Figure 1: Biot attenuation (inverse quality factor) and dispersion for porous rock containing $100 \%$ water (top) and heavy gas (bottom). Very modest amounts of attenuation and dispersion are predicted at high frequencies

Figure 2: Mesoscale distribution of two different pore fluids within a porous rock having lithological variations. The scale of fluid heterogeneities is greater then pore scale, but much less then wavelength scale.

Figure 3: Lower and upper bounds on $P$-wave velocities for partially water saturated porous rocks with light gas or heavy gas inclusions.

Figure 4: Periodic geometries (a) for 1d layers showing composite volume (b) for $3 \mathrm{~d}$, spherical inclusions are distributed in an array (c) the composite volume used to approximate the periodic array

Figure 5: (a) A random distribution of spherical inclusions of another poroelastic material. (b) a continuous random distribution of poroelastic materials

Figure 6: Shows the incident plane fast compressional wave and the reflected and refracted waves

Figure 7: Showing a compressional wave incident on a random distribution of spherical inclusions of another poroelastic material.

Figure 8: Attenuation due to mesoscopic fluid flow, given by White's concentric sphere model and macroscopic flow (Biot theory using Wood's law to define an effective fluid bulk modulus). $15 \%$ air inclusions within a water saturated porous rock of porosity $15 \%$ is modelled.

Figure 9: Attenuation estimates modelled using the periodic models of White [7], Johnson [8] and Pride et al [9]. Very good agreement between all approaches for the case of $5 \%$ air inclusions in an otherwise water saturated host rock of porosity $15 \%$. The inclusion radius is $25 \mathrm{~cm}$.

Figure 10: Phase velocity dispersion modelled using the periodic models of White [7], Johnson [8] and Pride et al [9]. Very good agreement between all approaches for the case of $5 \%$ air inclusions in an otherwise water saturated host rock of porosity $15 \%$. The inclusion radius is $25 \mathrm{~cm}$.

Figure 11: Attenuation and dispersion estimates from heavy gas inclusions within an otherwise water saturated porous rock of porosity 0.08 . White's model $(-)$, Ciz and Gurevich (--) (a) has an inclusion concentration of $0.1 \%$ (b) has an inclusion 
concentration of $10 \%$. (c) attenuation and dispersion when the more compressible fluid is modelled as the host saturating fluid. In this case, water inclusions are modelled within an air saturated host rock. Good agreement is seen between periodic and random estimates.

Figure 12: Attenuation and phase velocity dispersion estimates for the periodic model of White 1975 and the random continuous model. White's model (-), Toms et al 2005 (--) (a) has $35 \%$ air (b) has $35 \%$ of heavy gas, within an otherwise water saturated porous rock of porosity $12 \%$.

Figure 13: Shows attenuation estimates of the CRM model and the periodic reference model of White [7] against the experimental data of Murphy [84]. The CRM models produce closer estimates of attenuation then does the reference periodic model. 

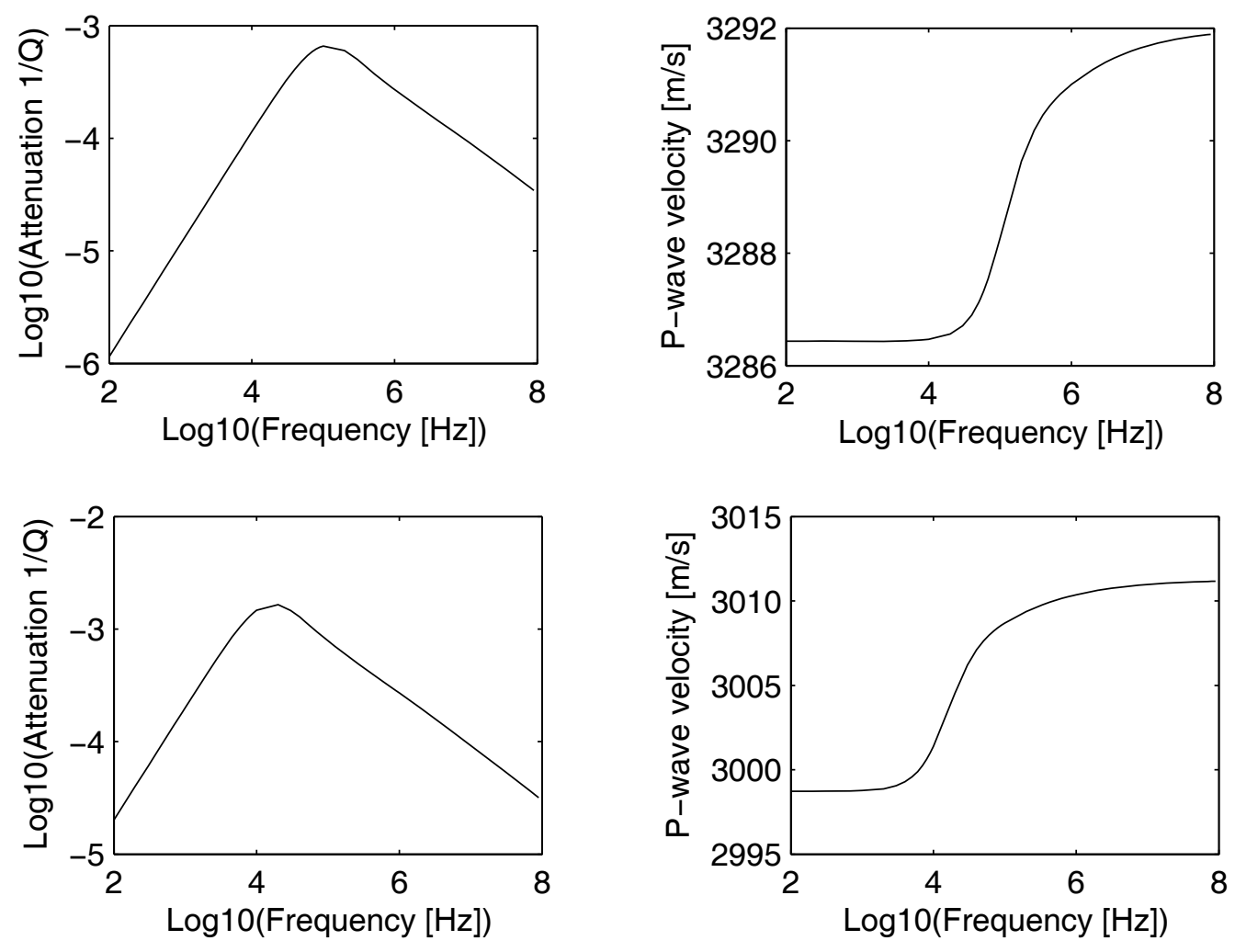

Figure 1: Biot attenuation (inverse quality factor) and dispersion for porous rock containing $100 \%$ water (top) and heavy gas (bottom). Very modest amounts of attenuation and dispersion are predicted at high frequencies 

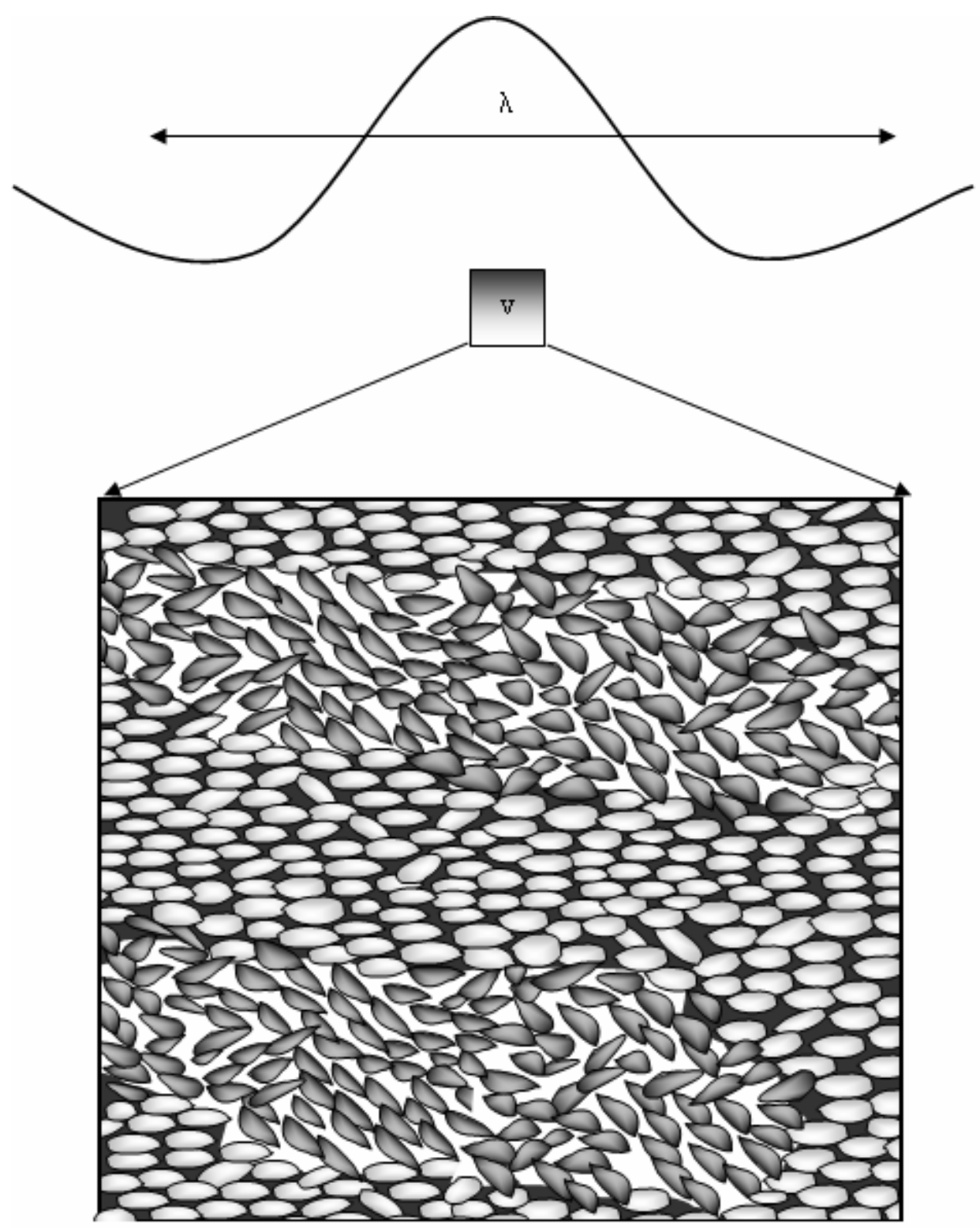

Figure 2: Mesoscale distribution of two different pore fluids within a porous rock having lithological variations. The scale of fluid heterogeneities is greater then pore scale, but much less then wavelength scale 


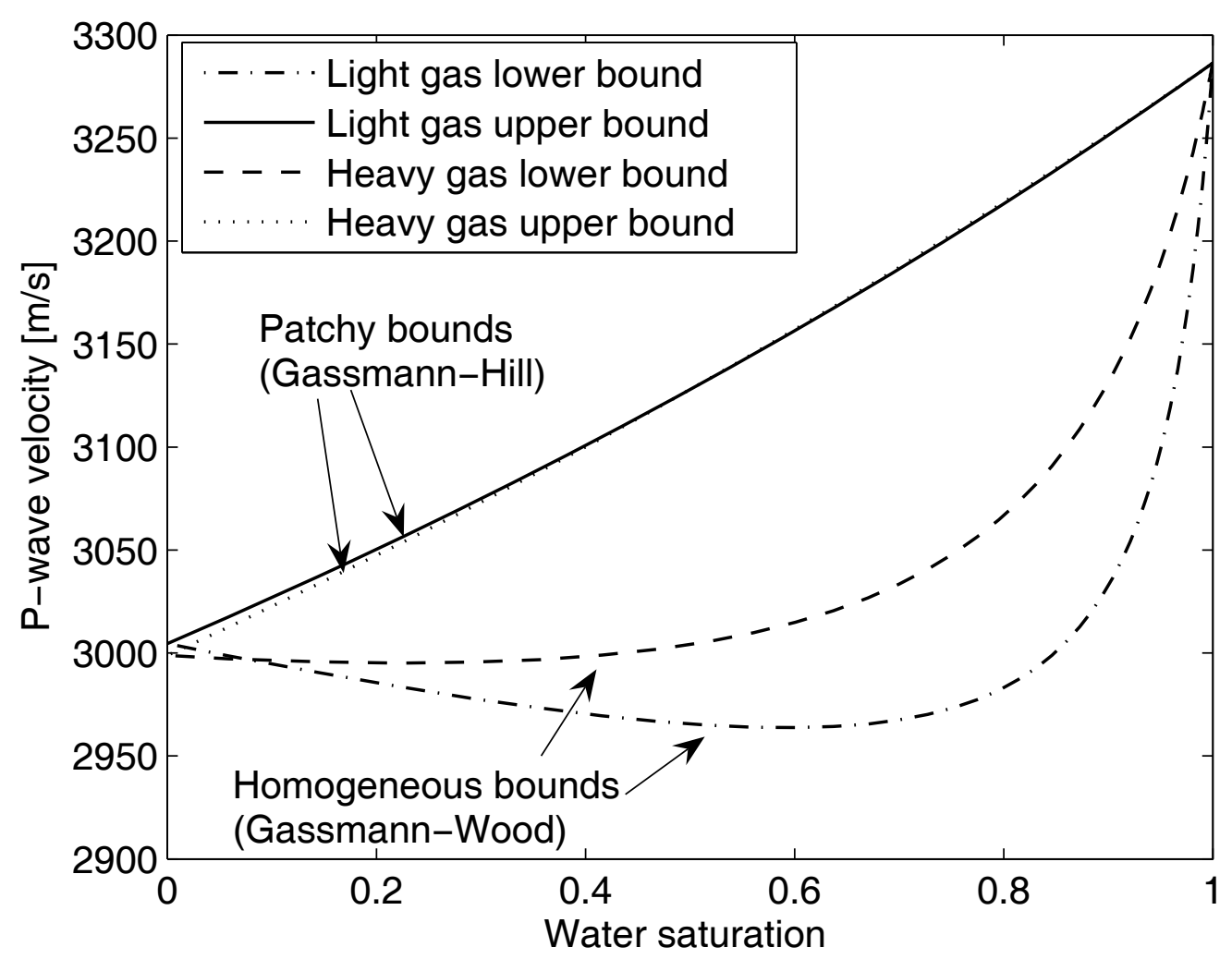

Figure 3: Lower and upper bounds on $P$-wave velocities for partially water saturated porous rocks with light gas or heavy gas inclusions. 


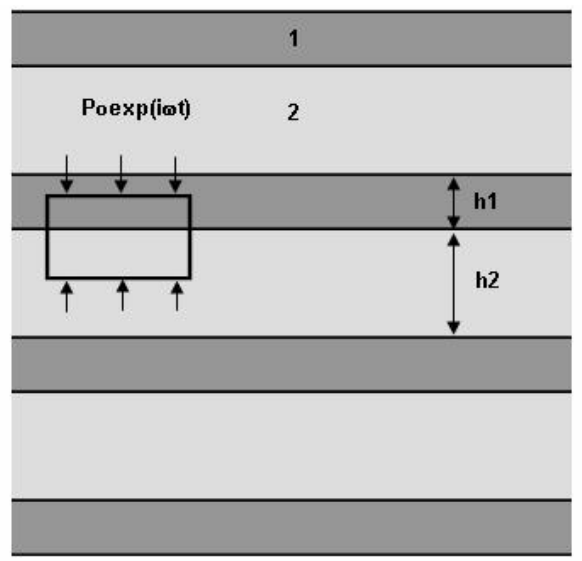

b)

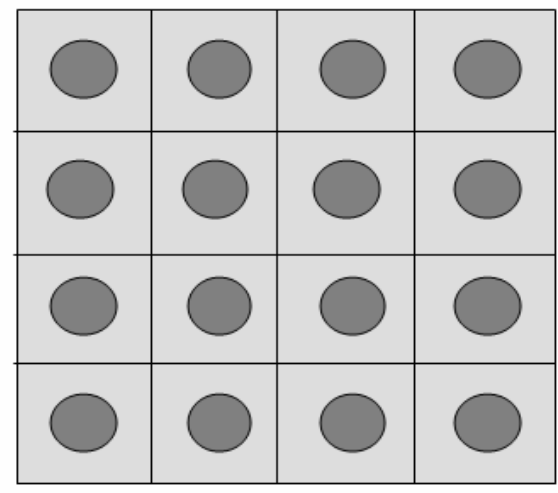

c)

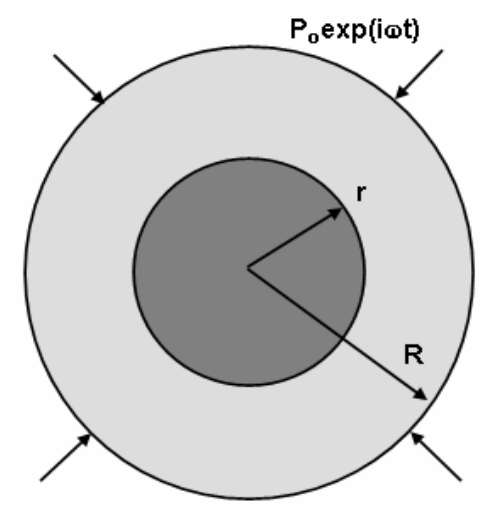

Figure 4: Periodic geometries (a) for 1d layers showing composite volume (b) for 3d, spherical inclusions are distributed in an array (c) the composite volume used to approximate the periodic array 
a)

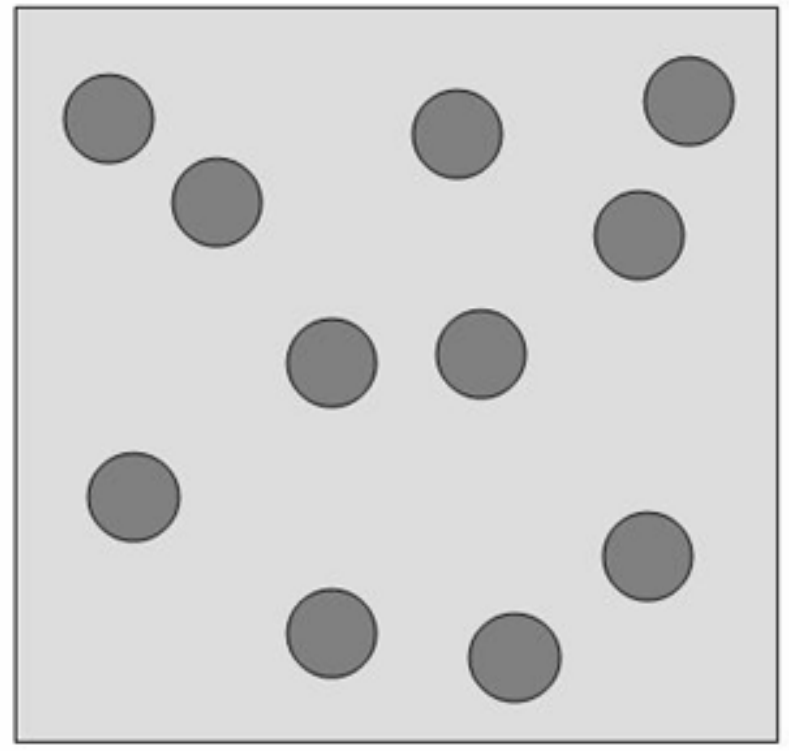

b)

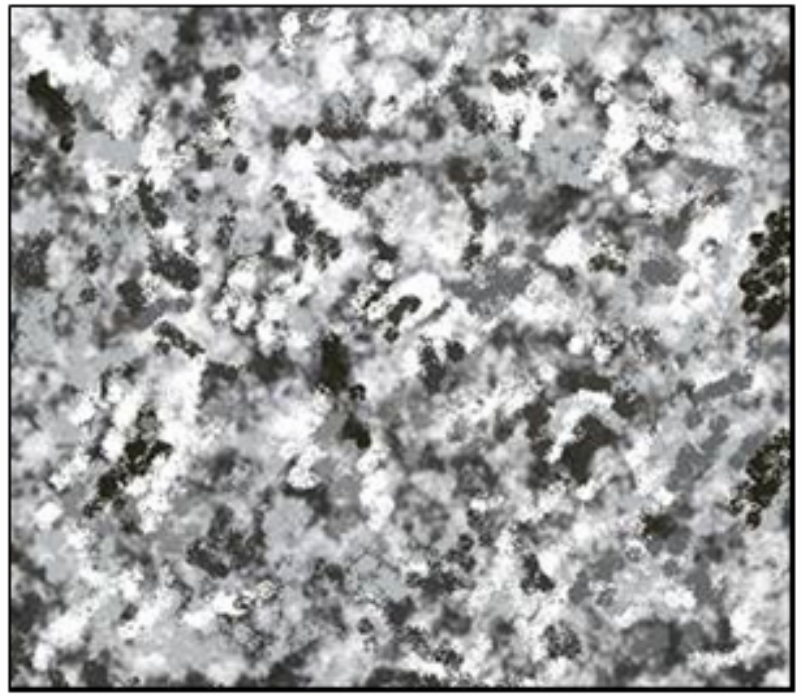

Figure 5: (a) A random distribution of spherical inclusions of another poroelastic material. (b) a continuous random distribution of poroelastic materials 


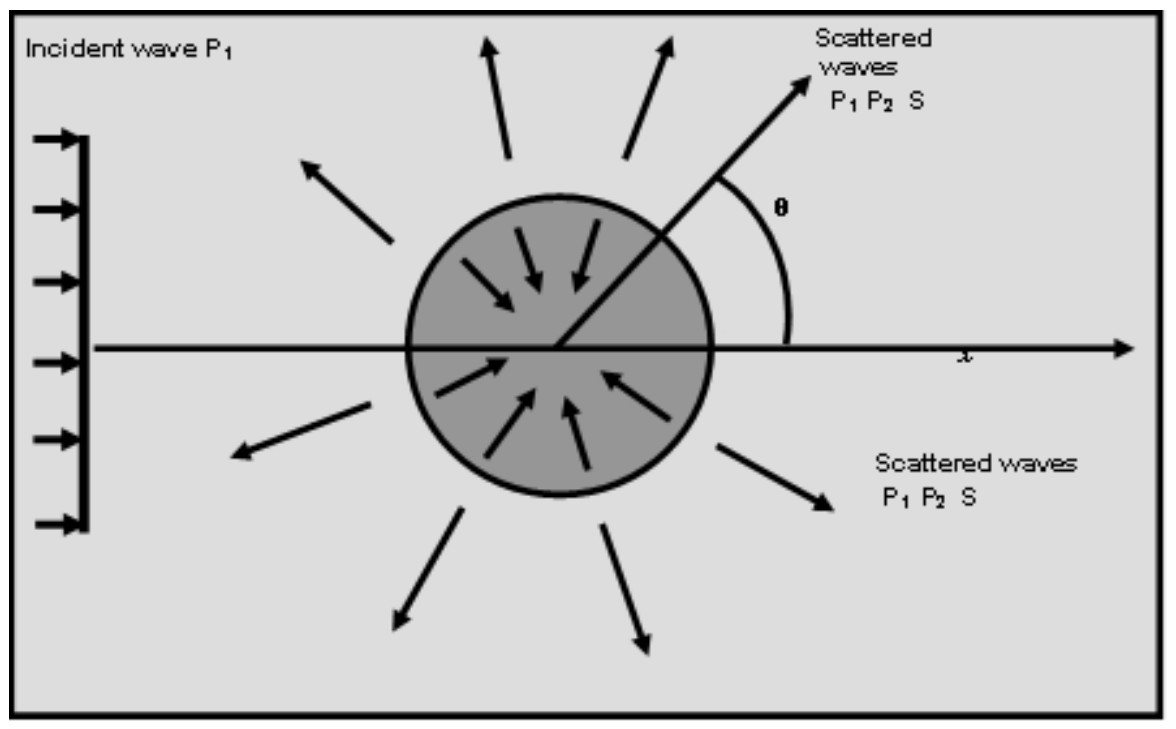

Figure 6: Shows the incident plane fast compressional wave and the reflected and refracted waves 


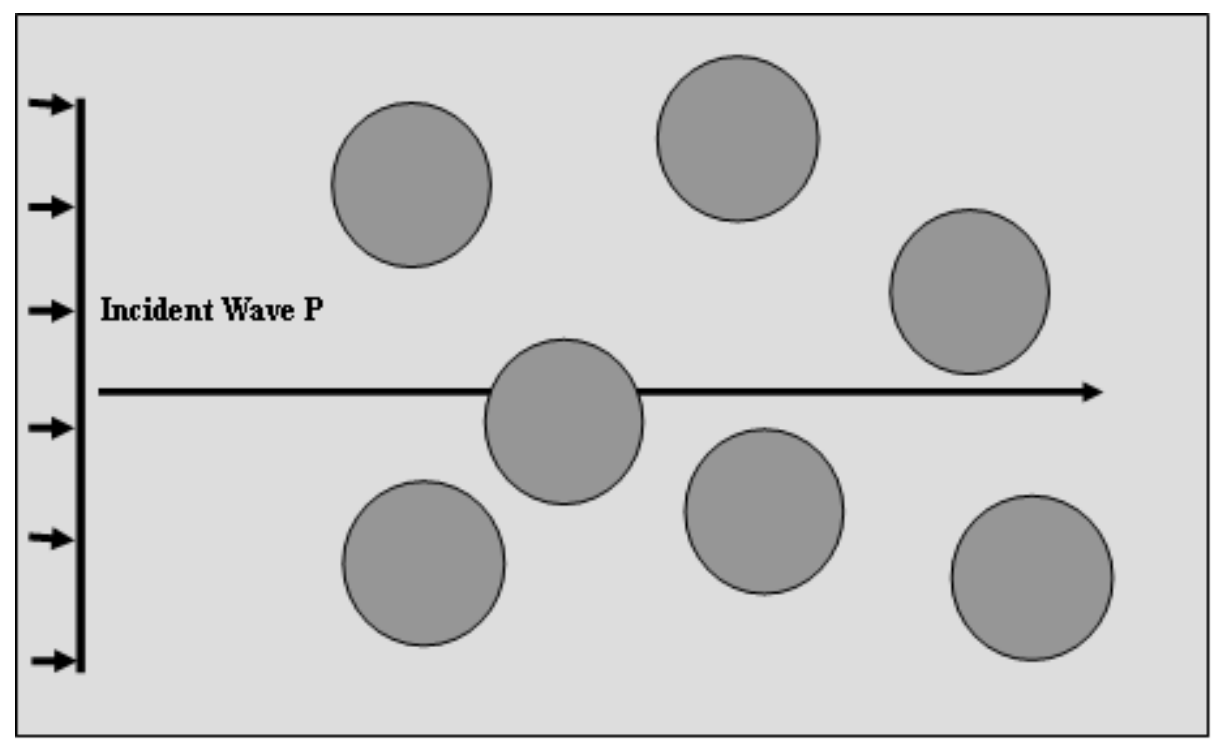

Figure 7: Showing a compressional wave incident on a random distribution of spherical inclusions of another poroelastic material. 


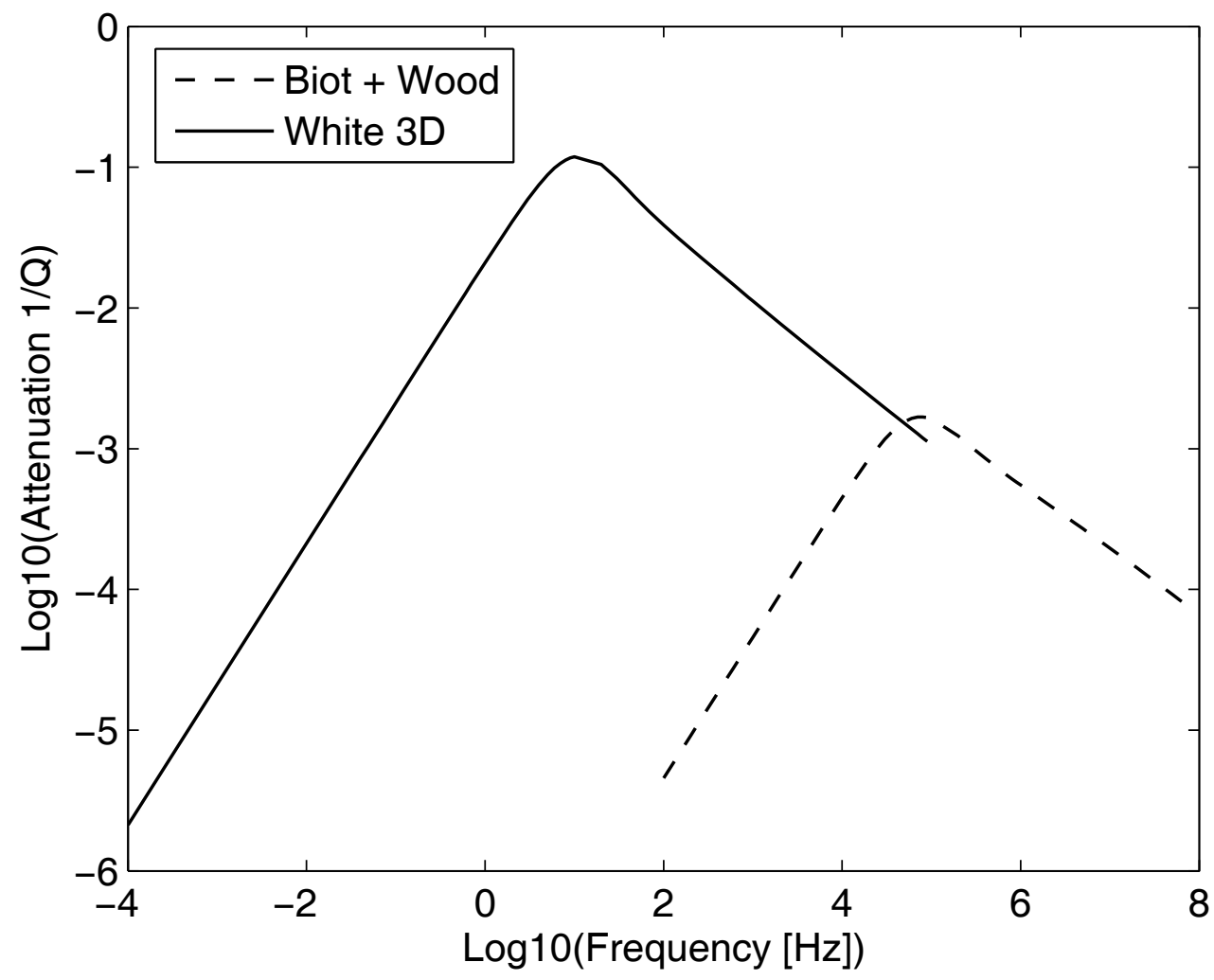

Figure 8: Attenuation due to mesoscopic fluid flow, given by White's concentric sphere model and macroscopic flow (Biot theory using Wood's law to define an effective fluid bulk modulus). $15 \%$ air inclusions within a water saturated porous rock of porosity $15 \%$ is modelled. 


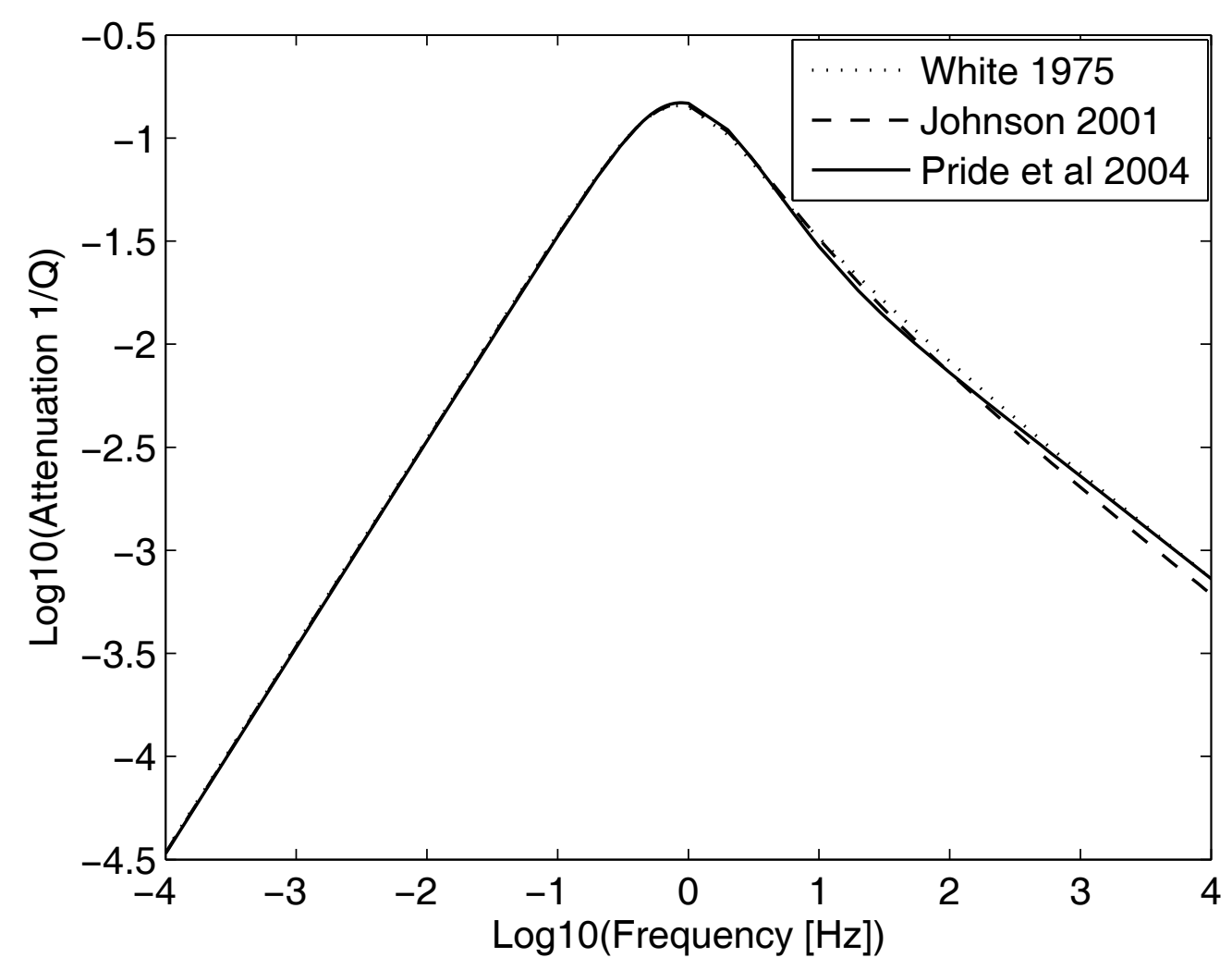

Figure 9: Attenuation estimates modelled using the periodic models of White [7], Johnson [8] and Pride et al [9]. Very good agreement between all approaches for the case of $5 \%$ air inclusions in an otherwise water saturated host rock of porosity $15 \%$. The inclusion radius is $25 \mathrm{~cm}$. 


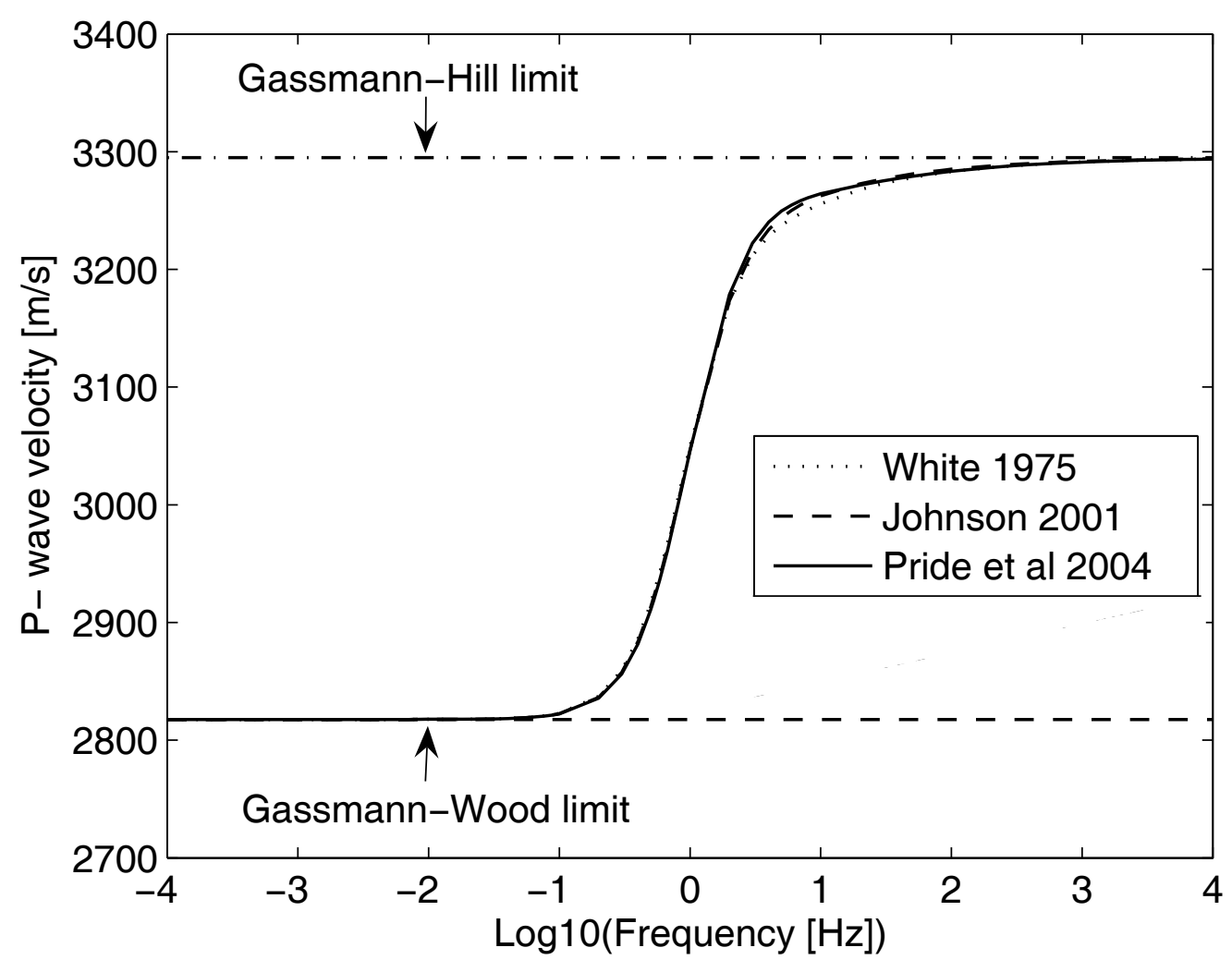

Figure 10: Phase velocity dispersion modelled using the periodic models of White [7], Johnson [8] and Pride et al [9]. Very good agreement between all approaches for the case of $5 \%$ air inclusions in an otherwise water saturated host rock of porosity $15 \%$. The inclusion radius is $25 \mathrm{~cm}$. 
a)

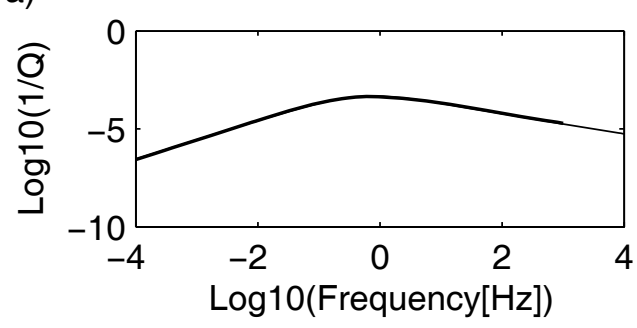

b)

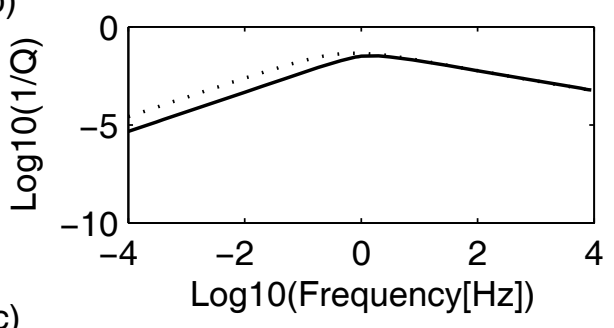

c)

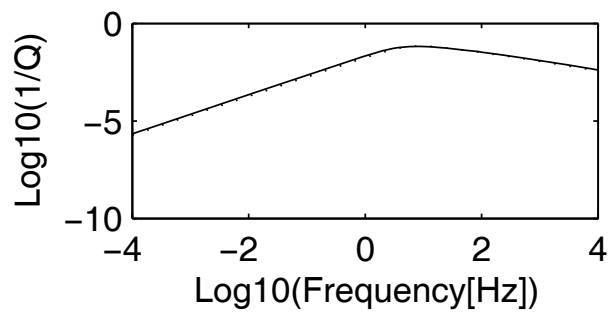

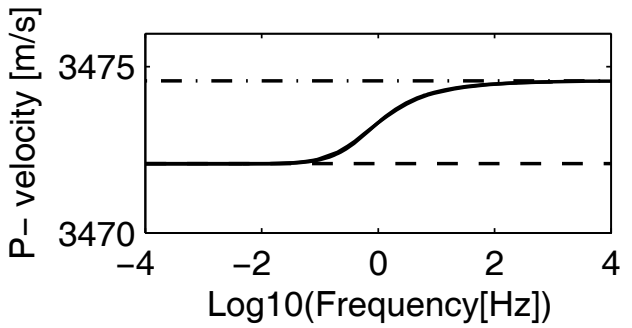
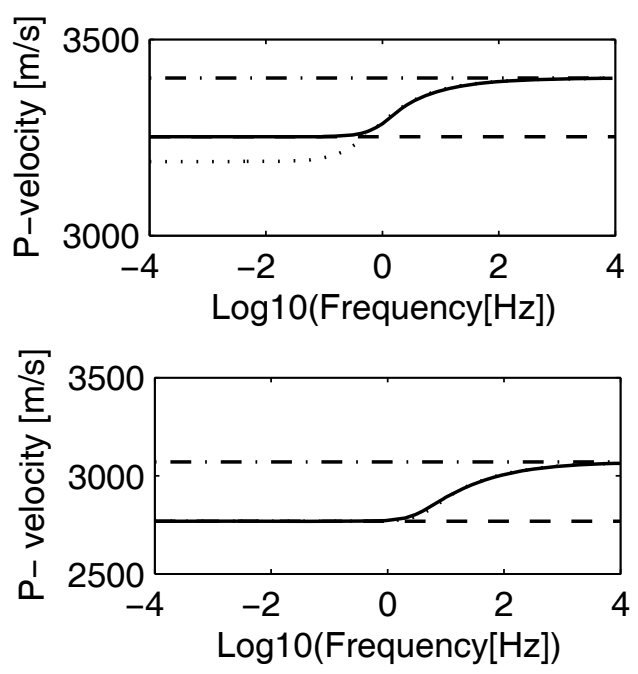

Figure 11: Attenuation and dispersion estimates from heavy gas inclusions within an otherwise water saturated porous rock of porosity 0.08 . White's model (-), Ciz and Gurevich (--) (a) has an inclusion concentration of $0.1 \%$ (b) has an inclusion concentration of $10 \%$. (c) attenuation and dispersion when the more compressible fluid is modelled as the host saturating fluid. In this case, water inclusions are modelled within an air saturated host rock. Good agreement is seen between periodic and random estimates. 
a)
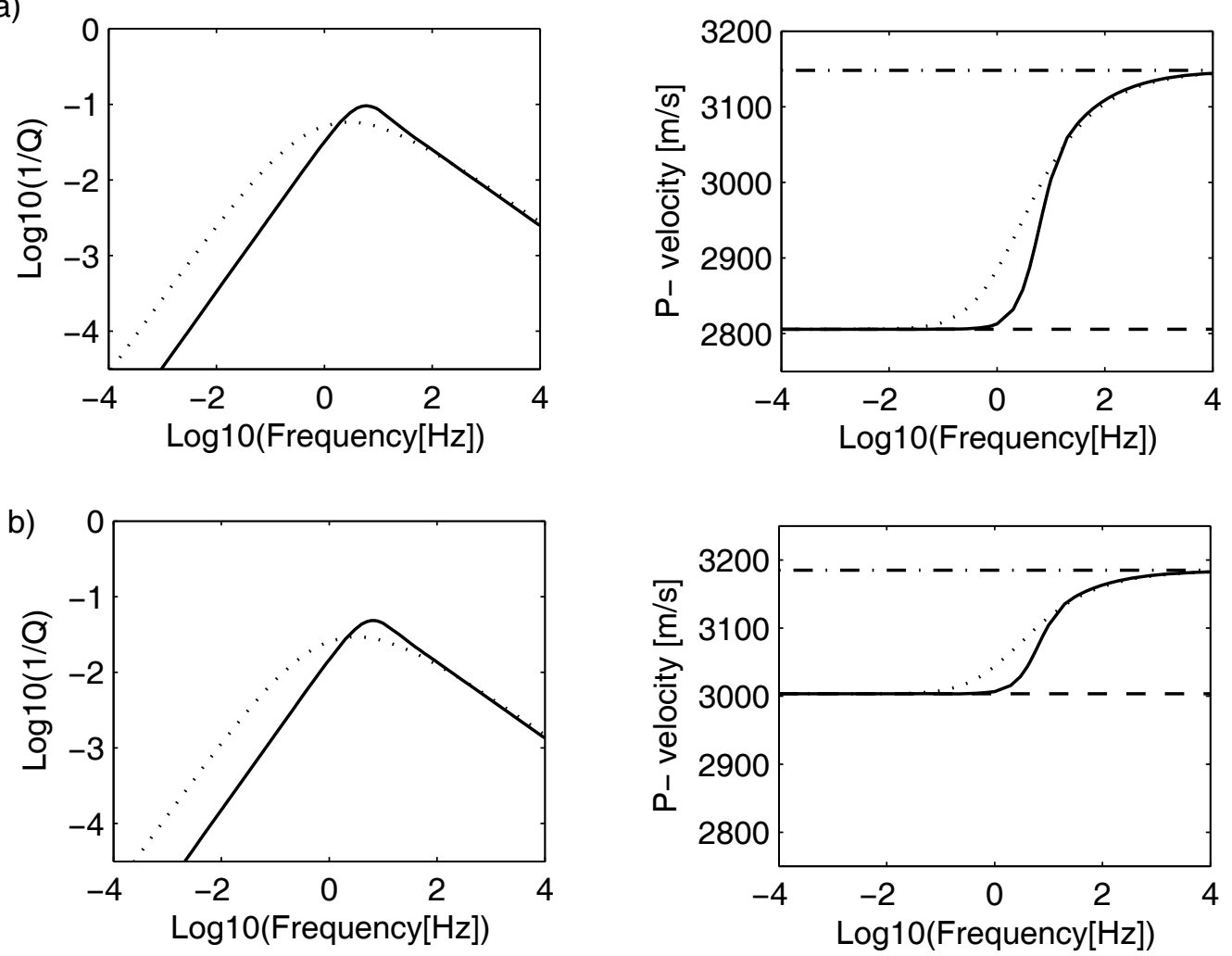

Figure 12: Attenuation and phase velocity dispersion estimates for the periodic model of White 1975 and the random continuous model. White's model (-), Toms et al 2005 (--) (a) has $35 \%$ air (b) has $35 \%$ of heavy gas, within an otherwise water saturated porous rock of porosity $12 \%$. 


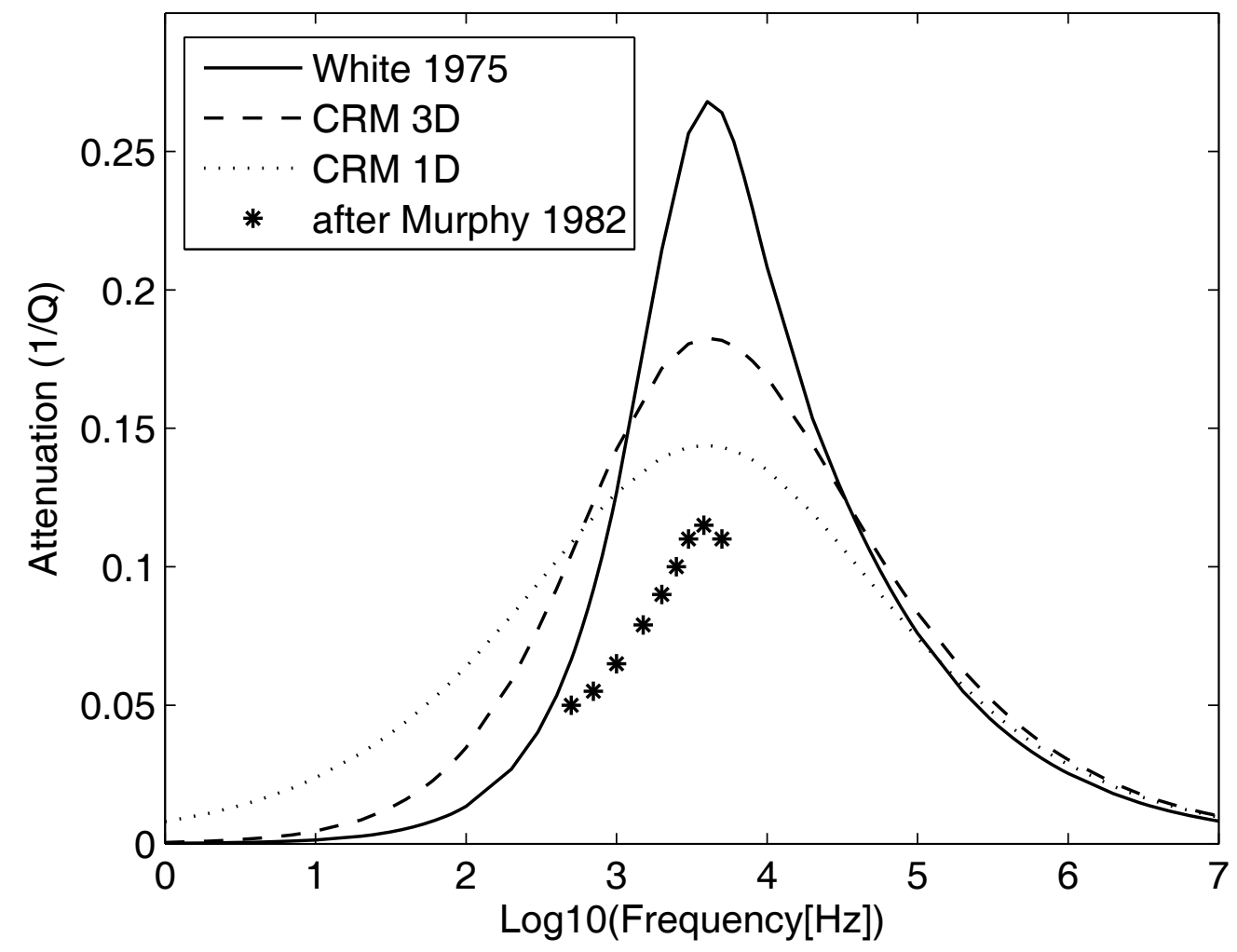

Figure 13: Shows attenuation estimates of the CRM model and the periodic reference model of White [7] against the experimental data of Murphy [84]. The CRM models produce closer estimates of attenuation then does the reference periodic model. 
Table List:

Table 1: shows the physical properties used in modelling attenuation and phase velocities 
Table 1: shows the physical properties used in modelling attenuation and phase velocities

\begin{tabular}{|c|c|c|c|c|c|}
\hline Rock & & Fluids & & Fluids & \\
\hline K & $\begin{array}{l}\text { GPa } \\
\text { GP }\end{array}$ & $\begin{array}{l}\mathbf{K}_{\mathrm{fw}} \\
\text { water }\end{array}$ & $\begin{array}{l}2.25 \\
\text { GPa }\end{array}$ & $\begin{array}{l}K_{f} \\
\text { h. gas }\end{array}$ & $\begin{array}{l}0.25 \\
\text { GPa }\end{array}$ \\
\hline$\mu$ & $\begin{array}{l}9 \\
\text { GPa }\end{array}$ & $\rho_{w}$ & $\begin{array}{l}990 \\
\mathrm{Kg} / \mathrm{m}^{3}\end{array}$ & $\rho$ & $\begin{array}{l}400 \\
\mathrm{Kg} / \mathrm{m}^{3}\end{array}$ \\
\hline$K_{g}$ & $\begin{array}{l}55 \\
\text { GPa }\end{array}$ & $\eta_{w}$ & $\begin{array}{l}\text { 1e-3 } \\
\text { Pa s }\end{array}$ & $\eta$ & $\begin{array}{l}\text { 6e-5 } \\
\text { Pa s }\end{array}$ \\
\hline$\rho_{\mathrm{g}}$ & $\begin{array}{l}2650 \\
\mathrm{Kg} / \mathrm{m}^{3}\end{array}$ & $\begin{array}{l}\mathbf{K}_{\mathrm{fa}} \\
\text { air }\end{array}$ & $\begin{array}{l}\text { 0.1 } \\
\text { MPa }\end{array}$ & $\begin{array}{l}K_{f} \\
\text { l. gas }\end{array}$ & $\begin{array}{l}\text { 0.1 } \\
\text { GPa }\end{array}$ \\
\hline$\phi$ & Varying & $\rho_{\mathrm{ga}}$ & $\begin{array}{l}100 \\
\mathrm{Kg} / \mathrm{m}^{3}\end{array}$ & $\rho_{\mathrm{g}}$ & $\begin{array}{l}100 \\
\mathrm{Kg} / \mathrm{m}^{3}\end{array}$ \\
\hline$\kappa$ & $\begin{array}{l}1 \mathrm{e}-13 \\
\mathbf{m}^{2}\end{array}$ & $\eta_{2 a}$ & $\begin{array}{l}\text { 1e-5 } \\
\text { Pa s }\end{array}$ & $\eta_{2}$ & $\begin{array}{l}\text { 3e-5 } \\
\text { Pa s }\end{array}$ \\
\hline
\end{tabular}

